\title{
Differential Effects of Cholesterol, Ergosterol and Lanosterol on a Dipalmitoyl Phosphatidylcholine Membrane: A Molecular Dynamics Simulation Study
}

\author{
Zoe Cournia, ${ }^{\dagger}$ G. Matthias Ullmann, ${ }^{\dagger, *}$ and Jeremy C. Smith $*, \dagger, \S$ \\ Computational Molecular Biophysics, Interdisciplinary Center for Scientific Computing (IWR), Im Neuenheimer \\ Feld 368, Universität Heidelberg, 69120 Heidelberg, Germany, Structural Biology and Bioinformatics, \\ University of Bayreuth, Universitätsstrasse 30, Gebäude B14, 95447 Bayreuth, Germany, and Oak Ridge \\ National Laboratory and Center for Molecular Biophysics, University of Tennessee, 1 Bethel Valley Road, \\ Oak Ridge, Tennessee 37831
}

Received: August 10, 2006; In Final Form: November 13, 2006

\begin{abstract}
Lipid raft/domain formation may arise as a result of the effects of specific sterols on the physical properties of membranes. Here, using molecular dynamics simulation, we examine the effects of three closely-related sterols, ergosterol, cholesterol, and lanosterol, at a biologically relevant concentration (40 mol \%) on the structural properties of a model dipalmitoyl phosphatidylcholine (DPPC) membrane at 309 and $323 \mathrm{~K}$. All three sterols are found to order the DPPC acyl tails and condense the membrane relative to the DPPC liquidphase membrane, but each one does this to a significantly different degree. The smooth $\alpha$-face of ergosterol, together with the presence of tail unsaturation in this sterol, leads to closer interaction of ergosterol with the lipids and closer packing of the lipids with each other, so ergosterol has a higher condensing effect on the membrane, as reflected by the area per lipid. Moreover, ergosterol induces a higher proportion of trans lipid conformers, a thicker membrane, and higher lipid order parameters and is aligned more closely with the membrane normal. Ergosterol also positions itself closer to the bilayer/water interface. In contrast, the rough $\alpha$-face of lanosterol leads to a less close interaction of the steroid ring system with the phospholipid acyl chains, and so lanosterol orders, straightens, and packs the lipid acyl chains less well and is less closely aligned with the membrane normal. Furthermore, lanosterol lies closer to the relatively disordered membrane center than do the other sterols. The behavior of cholesterol in all the above respects is intermediate between that of lanosterol and ergosterol. The findings here may explain why ergosterol is the most efficient of the three sterols at promoting the liquid-ordered phase and lipid domain formation and may also furnish part of the explanation as to why cholesterol is evolutionarily preferred over lanosterol in higher-vertebrate plasma membranes.
\end{abstract}

\section{Introduction}

The physical and chemical properties of biological membranes are of critical importance for understanding specific membrane function. Membranes of eukaryotic cells have complex compositions consisting of hundreds of different lipids, proteins, and sterols. Among the sterols, cholesterol is particularly abundant and has been found to account for up to $50 \%$ of the lipid concentration in the plasma membrane of higher vertebrates. ${ }^{1}$

The effect of cholesterol on functional, structural, and dynamical membrane properties has received considerable attention in the past decades. ${ }^{2}$ Cholesterol has been shown to alter the bulk biophysical properties of membranes, such as their fluidity, 3,4 and may also play other membrane-associated roles, such as regulating the membrane proteins function ${ }^{5}$ and influencing membrane permeability. ${ }^{6}$ Other biologically important sterols include ergosterol (provitamin $\mathrm{D}_{2}$ ), which is found in the membranes of fungi, yeasts, and protozoans, and lanosterol, the evolutionary and biosynthetic precursor of cholesterol, ${ }^{7}$ which is the major constituent of prokaryotic cell membranes.

* Corresponding author. Tel: +496221548857. Fax: +496221548868. E-mail: biocomputing@iwr.uni-heidelberg.de.

$\dagger$ IWR.

Structural Biology and Bioinformatics.

$\S$ Oak Ridge National Laboratory and University of Tennessee.
Cholesterol, ergosterol, and lanosterol have very similar chemical structures (see Figure $1 b-d$, respectively). The main structural difference between lanosterol and the other two sterols is the presence of three extra methyl groups (two at position $\mathrm{C}_{4}$ and one at $\mathrm{C}_{14}$ ) that protrude from its otherwise flat $\alpha$-surface. In the steroid ring system, cholesterol has a double bond between carbons $\mathrm{C}_{5}$ and $\mathrm{C}_{6}$, in contrast to lanosterol's double bond between $\mathrm{C}_{8}$ and $\mathrm{C}_{9}$. Ergosterol has a carbon-carbon double bond conjugated system in its second steroid ring, which has two cis hydrogens bound to $\mathrm{C}_{6}$ and $\mathrm{C}_{7}$. Small chemical differences are also present in the alkyl side chain. Cholesterol has a saturated side chain, whereas ergosterol has a trans double bond at position $\mathrm{C}_{22}$ and is methylated at position $\mathrm{C}_{24}$, and lanosterol has a double bond at position $\mathrm{C}_{24}$. Although the above differences seem to be subtle, only cholesterol has been chosen by evolution to be a major constituent of the higher vertebrate plasma membranes. Furthermore, the conversion of lanosterol to cholesterol in vertebrate cells is a long, complicated, and energetically expensive path, requiring 18 enzymatic steps. ${ }^{7}$ Depletion of cholesterol or its substitution with ergosterol or lanosterol is lethal to all mammalian cells. Accordingly, cholesterol cannot substitute for ergosterol in yeast.

Lipid bilayers composed of a single phospholipid species undergo a well-defined phase transition in which the lipid chains change from an ordered or gel state to a fluid or liquid crystalline 
state. Upon addition of cholesterol in a concentration above 25 mol \%, there is a dramatic influence on the gel-to-liquid crystal lipid-phase transition. A new thermodynamically stable region of coexistence between the liquid-disordered ("Id") and solidordered ("so") or gel phase is described: the liquid-ordered ("lo") phase. ${ }^{8-13}$ This new phase is characterized by a fluidity intermediate between those of the gel and the fluid phases formed by the pure lipids. Recently, it has been proposed that the lo phase is formed when cholesterol associates with saturated, high-melting lipids, such as DPPC and sphingomyelin, to create dynamic complexes in model membranes, so-called "lipid rafts". Cholesterol promotes a phase separation in model membranes where cholesterol-rich and cholesterol-poor microdomains are formed. ${ }^{14-18}$

Evidence that ergosterol is also able to promote the formation of the lo phase was very recently published. ${ }^{19,20}$ A fluorescence study showed that ergosterol promotes domain formation more strongly than cholesterol. ${ }^{21}$ The DPPC-ergosterol phase diagram is very similar to that obtained for DPPC-cholesterol, but to achieve the formation of the lo phase in the ergosterolDPPC membrane, a concentration of ergosterol above $30 \mathrm{~mol}$ $\%$ is needed. ${ }^{19}$

Lanosterol is less prone to inducing the lo phase than ergosterol or cholesterol and has little effect on domain formation. ${ }^{11,22,23}$ In cholesterol-containing membranes, the lo phase is a well-defined thermodynamic phase, clearly separated from the ld phase. In contrast, in lanosterol-containing membranes, calculated and experimental phase diagrams show, that the lo and ld phases are no longer thermodynamically distinguishable. ${ }^{11,22}$

The above discussion indicates that, although cholesterol, ergosterol, and lanosterol have very similar chemical structures, their effects on lipid membranes are very different. The way in which cholesterol and ergosterol promote raft formation, whereas lanosterol has limited capacity, must lie in the way these sterols modulate the physical properties of membranes and, thus, in the specific sterol/saturated lipid interactions in the membrane. The specific chemical structures of these molecules, also in comparison to lanosterol, will be the cause of the effects that are observed in sterol-containing lipid membranes, such as lipid ordering and the lo phase formation. An intriguing question is which are the particular chemical characteristics of cholesterol that have led to its being evolutionarily selected for higher vertebrate plasma membranes, given its structural similarity to its precursors.

A possible argument for the evolutionary preference for cholesterol might lie in cholesterol's ability to promote lipid raft formation better than lanosterol. ${ }^{11,22,23}$ Lipid rafts (i.e., cholesterol-rich lipid domains), which are postulated to exist in mammalian plasma membranes, have been extensively researched in the past few years, partly because they have changed the traditional notion of the homogeneous fluid mosaic model of the plasma membrane and also because of their possible roles in HIV, Alzheimer, and prion diseases. ${ }^{24,25}$ The possibility of modulating lipid raft properties using statins and sterol/synthetic sphingolipid analogues opens up new approaches for therapeutic interventions in such raft-associated diseases.

Although the definition of the lo phase is now established, ${ }^{2}$ a number of questions associated with the characteristics of this phase are still being debated. For example, there is little information on the position of cholesterol/ergosterol in the membrane, the role of hydrogen bonding, the population of gauche lipid conformers, the dynamics of the sterols in the rafts, and the origin of the inequivalence of the interaction between the sterols and the lipid acyl sn1 and the sn2 chains.

There has been much interest in studying the differences in structure and dynamics between biologically important sterols (e.g., cholesterol, ergosterol, lanosterol) in membranes. ${ }^{19,23,26-29}$ Among them, the relevant questions are whether the different sterol molecules interact differently with the same lipid and whether the different sterols have different dynamics in the membrane. A variety of experimental and molecular dynamics (MD) studies have also attempted to investigate the diffusion of sterols in phospholipid model membranes. ${ }^{30-37}$

Physical properties of mammalian plasma membranes can be reproduced and studied with model biological membranes. ${ }^{2}$ Both experimental and computational studies of model systems have shed light on the nature of phospholipid-sterol interactions. Although valuable, experimental studies aimed at determining how sterols influence membrane properties very often lack sufficient resolution for investigating the detailed underlying molecular interactions involved. In contrast, using MD simulations, it is possible to interpret experimental results on complex membrane systems in detail and to gain insight into the relevant interactions at the atomic level. The field of lipid bilayer simulation is growing rapidly and with it, the level of complexity of the systems, with explicit inclusion of membrane proteins ${ }^{38-42}$ and cholesterol ${ }^{43-46}$ in the simulated systems. Although several MD simulations of lipid/cholesterol systems have been published over the past decade, ${ }^{34,35,43-54}$ only two have investigated the different effects of various sterols in the membrane. ${ }^{43,55}$

In the present paper, cholesterol, ergosterol, and lanosterol are studied in DPPC at two temperatures, chosen such that one is below $(T=309 \mathrm{~K})$ and one is above $(T=323 \mathrm{~K})$ the DPPC gel-liquid transition, which occurs at $T=315 \mathrm{~K}$ for the pure DPPC system. ${ }^{56}$ Simulations of pure DPPC in the gel (309 K) and the liquid $(323 \mathrm{~K})$ phases have also been performed as controls. For the liquid DPPC membrane at $T=323 \mathrm{~K}$, the effect of surface tension application on the bilayer was also studied.

This MD study of cholesterol-, ergosterol-, and lanosterolDPPC membranes examines a variety of sterol-DPPC membrane properties at $40 \mathrm{~mol} \%$ concentration and at two different temperatures. The results demonstrate that the small differences in the chemical structure of the three sterols give rise to significant differences in their effects on the membrane properties. The position of each sterol, the population of gauche conformers, and the ordering effect on the DPPC acyl chains, the area per lipid, the hydration levels of each sterol, and the inequivalence of the interaction between the sterols and the sn1 and sn 2 DPPC acyl chains in the membrane are examined. The present study also points out which particular chemical characteristics of these three molecules are responsible for the differences in membrane properties. The results provide insight as to why ergosterol is the strongest promoter of the formation of the lo phase among the three sterols studied and lanosterol is the weakest. Moreover, the comparison between lanosterol and cholesterol may shed light onto why the latter is evolutionarily preferred for the higher vertebrate plasma membranes.

\section{Methods}

Computational Details. The simulations were performed at $T=309$ and $323 \mathrm{~K}$ on cholesterol/DPPC, ergosterol/DPPC and lanosterol/DPPC bilayers, together with "control" simulations of pure DPPC. For all simulations, the CHARMM package version $28 \mathrm{~b} 1^{57}$ was used with the all-atom CHARMM 27 force field ${ }^{57}$ for DPPC. For cholesterol, ergosterol, and lanosterol, the force field derived in ref 58 was used. 
The Particle Mesh Ewald summation technique ${ }^{59}$ was employed to calculate electrostatic contributions. The van der Waals and electrostatic interactions were cut off at $14 \AA$ using the CHARMM shifted potential. It has been shown that shifting is an appropriate method of electrostatic truncation in lipid bilayer simulations. ${ }^{60}$ Periodic boundary conditions were applied to the orthorhombic cells in all directions. The equation of motion was integrated using the Verlet algorithm ${ }^{61}$ with a time step of $1 \mathrm{fs}$. The simulations were carried out in the $N P_{\mathrm{n}} \gamma T$ ensemble (constant number of molecules, normal pressure, surface tension, and temperature), in which the surface tension and the normal pressure are specified. The surface tension, $\gamma$, is defined as ${ }^{62}$

$$
\gamma=\int_{-\infty}^{\infty} \mathrm{d} z\left[P_{\mathrm{n}}-P_{1}(z)\right]
$$

where $P_{\mathrm{n}}$ is the normal component and $P_{1}$ is the lateral pressure of the pressure tensor. The box length in the $z$ direction was allowed to vary independently of $x$ and $y$ and was coupled to the $z$ component of the pressure tensor. The $x$ and $y$ dimensions of the unit cell vary while maintaining constant surface tension. ${ }^{63}$ In the case that the average lateral pressure and the normal pressures are equal, the surface tension is zero. The values of the surface tension chosen for this study depend on the system being simulated: the test calculations for this are described in the Appendix.

A constant pressure of $P=1$ atm was imposed using the Langevin piston method. ${ }^{64}$ The collision frequency was set to $30 \mathrm{ps}^{-1}$ for the heating and equilibration and to $10 \mathrm{ps}^{-1}$ for production dynamics. Constant temperature conditions were maintained by a Hoover thermostat ${ }^{65}$ using the extended system constant pressure and temperature algorithms implemented in CHARMM with a mass of $2000 \mathrm{kcal} / \mathrm{ps}$.

Before analysis, all coordinate sets were superposed on a primary-box reference structure in order to remove global unitcell rotation and translation. Analysis of the system properties was performed using a combination of CHARMM utilities and our own analysis code. The CPU time needed for the production runs of all systems was $11360 \mathrm{~h}$ on 16 Dual AMD Athlon 1.4 Ghz processors with a Myrinet 2 Gbit network. The total time of the simulations presented here is over $150 \mathrm{~ns}$.

Control Simulations. For DPPC, the gel-to-liquid-phase transition occurs at $T_{\mathrm{m}}=315 \mathrm{~K} .{ }^{56}$ At $309 \mathrm{~K}$, the DPPC membrane is below its main phase transition and is found in a gel state. Above the transition temperature (e.g., at $323 \mathrm{~K}$ ), the DPPC membrane is found in the biologically important liquid phase. These temperatures were chosen in order to study the sterol-containing membranes in comparison with the gel and the liquid phases and to evaluate the effect of the temperature change on these systems.

Gel DPPC Membrane. For the gel DPPC system, two simulations, one with 64 lipids and 762 water molecules and one with 256 lipids and 3048 water molecules (11.9 waters/ lipid), were performed. The initial configuration of the 64-lipid system was taken from the final frame of the NPT simulation in ref 66. The initial dimensions of the box were $x=42.79, y$ $=33.73$, and $z=64.90 \AA$. The 256-lipid unit cell was constructed by replicating the initial 64-lipid unit cell in the $x$ and $y$ axes, resulting in initial dimensions for this system of $x$ $=85.58, y=67.46$, and $z=64.90 \AA$. These two sizes were chosen to check for system size effects.

Simulation Protocol. For the simulation of DPPC in the gel phase, the protocol proposed in ref 66 was followed. Given that simulation at a constant area leads to a pleated structure ${ }^{66}$ which according to experimental evidence ${ }^{67}$ is an artifact, the $N P_{\mathrm{n}} \gamma T$ ensemble with $\gamma=0$ was chosen. The two membranes were minimized first for 100 steps with the steepest descent (SD) algorithm. Next, another 100 steps of conjugated gradient (CG) minimization was applied, followed by a final 9400 steps of the adopted basis Newton Raphson (ABNR) minimization with an exit criterion of an energy change of $<10^{-4} \mathrm{kcal} / \mathrm{mol}$. The systems were then heated to $309 \mathrm{~K}$ over $160 \mathrm{ps}$ in $2-\mathrm{K}$ increments. Equilibration followed for 50 ps with a $10-\mathrm{K}$ window for rescaling the velocities. Further equilibration without velocity rescaling was required for $1 \mathrm{~ns}$. The production run was then carried out for $4 \mathrm{~ns}$ for the 256 system and for $8 \mathrm{~ns}$ for the 64-lipid system.

Liquid DPPC Membrane. For the liquid DPPC system, again two simulations, one with 72 lipids and 2094 water molecules and one with 200 lipids and 5814 water molecules (29 waters/lipid), were performed at $T=323 \mathrm{~K}$. The initial configuration for the 72-lipid system was taken from the last frame of the 20-ns DPPC simulation performed in ref 68. The initial dimensions of this system were $x=47.58, y=47.58$ and $z=66.52 \AA$. The 200-lipid system was constructed by duplicating selected lipids in the $x$ and $y$ axes in order to yield the same system size as used in the sterol-DPPC simulations. The initial dimensions for this system were $x=67.50, y=$ 93.20 and $z=66.52 \AA$.

Simulation Protocol. The 72- and the 200-DPPC systems in the liquid phase were energy-minimized with 10000 steps of the SD algorithm. Next, another 20000 steps of CG minimization were performed, followed by 10000 steps of ABNR minimization with an exit criterion of an energy change of $<10^{-6} \mathrm{kcal} / \mathrm{mol}$. This energy tolerance was satisfied after 5000 steps. Subsequently, the systems were heated to $323 \mathrm{~K}$ over 65 ps in 5-K increments. Equilibration followed for $50 \mathrm{ps}$ with a $10-\mathrm{K}$ window for rescaling the velocities. Additional equilibration for $1 \mathrm{~ns}$ followed without rescaling the velocities. For the 72-DPPC system, the production runs were carried out for $2 \mathrm{~ns}$ for each of the surface tension values chosen (i.e., 0, $10,24.5,25,30$, and $61 \mathrm{dyn} / \mathrm{cm}$ ). For the optimal value found, $\gamma=24.5 \mathrm{dyn} / \mathrm{cm}$, the production run was continued up to $8 \mathrm{~ns}$. The choice of this value is described in the Appendix. The production run for the 200-DPPC system was carried out for 4 ns also at $\gamma=24.5 \mathrm{dyn} / \mathrm{cm}$.

A separate simulation of a pure water box at $T=309$ and $323 \mathrm{~K}$ with the same electrostatic treatment and simulation conditions as the lipid simulation (the pressure was the same in all three directions) was also performed in order to obtain an estimate of a water molecule volume, $V_{\mathrm{W}}$.

Sterol-DPPC Bilayer Systems. For the sterol-containing bilayer MD simulations, the membrane consisted of 120 DPPC and 80 sterol molecules, giving a biologically relevant $40 \mathrm{~mol}$ $\%$ sterol concentration. Six simulations of the lipid bilayer were performed with the three different sterols (cholesterol, ergosterol, and lanosterol) being simulated at two temperatures; namely, 309 and $323 \mathrm{~K}$. Coordinates for DPPC were constructed within CHARMM ${ }^{57,69}$ Selected DPPC molecules were replaced with cholesterol molecules in order to obtain a $40 \mathrm{~mol} \%$ sterol concentration, well within the lo phase. Coordinates for the cholesterol molecule were taken from its crystal structure. ${ }^{70}$ Since there is no consensus for a specific organization pattern of cholesterol in the membrane, in this simulation, the cholesterol molecules were roughly uniformly placed in the bilayer patch, with the same number of sterol molecules in each bilayer leaflet. The cholesterol hydroxyl group was placed at the same depth as the carbonyl group of DPPC, as suggested by structural 
TABLE 1: Average Cell Dimensions $x, y, z$; Cell Volume, $V_{\text {Tот }}$; Volume Per Lipid, $V_{\mathrm{DPPC}}$; Area per Lipid, $A_{\mathrm{DPPC}}$; and Membrane Thickness, $h$, for the Eight Systems and the Two Different Temperatures

\begin{tabular}{|c|c|c|c|c|c|c|c|c|}
\hline & \multicolumn{2}{|c|}{ DPPC-Chol } & \multicolumn{2}{|c|}{ DPPC-Erg } & \multicolumn{2}{|c|}{ DPPC-Lan } & \multicolumn{2}{|c|}{ pure $\mathrm{DPPC}^{a}$} \\
\hline & $309 \mathrm{~K}$ & $323 \mathrm{~K}$ & $309 \mathrm{~K}$ & $323 \mathrm{~K}$ & $309 \mathrm{~K}$ & $323 \mathrm{~K}$ & $309 \mathrm{~K}$ & $323 \mathrm{~K}$ \\
\hline$x, \AA$ & $55.4 \pm 0.3$ & $57.1 \pm 0.4$ & $56.7 \pm 0.4$ & $58.7 \pm 0.5$ & $56.9 \pm 0.4$ & $60.1 \pm 0.5$ & $89.3 \pm 0.6$ & $65.4 \pm 0.9$ \\
\hline$y, \AA$ & $71.5 \pm 0.3$ & 7 & $68.5 \pm 0.6$ & $67.2 \pm$ & $73.6 \pm 0.5$ & 71. & $66.3 \pm 0.2$ & 0.9 \\
\hline$z, \AA$ & $59.9 \pm 0.3$ & $59.3 \pm 0.4$ & $61.0 \pm 0.2$ & $60.7 \pm 0.3$ & $58.1 \pm 0.3$ & $57.1 \pm 0.4$ & $62.9 \pm 0.4$ & $67.1 \pm 1.0$ \\
\hline$V_{\mathrm{TOT}^{b}}, \AA^{3}$ & $237320 \pm 665$ & $240130 \pm 790$ & $236870 \pm 731$ & $239300 \pm 684$ & $243470 \pm 692$ & $246500 \pm 914$ & $372630 \pm 747$ & $414750 \pm 1071$ \\
\hline$V_{\text {DPPC }}, \AA^{3}$ & $1175.3 \pm 5.5$ & $1194.4 \pm 6.6$ & $1163.7 \pm 6.0$ & $1179.6 \pm 5.7$ & $1188.4 \pm 5.8$ & $1209.4 \pm 7.6$ & $1110.4 \pm 3.0$ & $1221.0 \pm 5.2$ \\
\hline$A_{\mathrm{DPPC}}, \AA^{2}$ & $48.3 \pm 0.2$ & $49.6 \pm 0.3$ & $47.1 \pm 0.2$ & $47.9 \pm 0.2$ & $49.8 \pm 0.2$ & $50.8 \pm 0.3$ & $46.8 \pm 0.2$ & $64.6 \pm 0.3$ \\
\hline$h, \AA$ & $48.7 \pm 0.5$ & $48.2 \pm 0.3$ & $49.4 \pm 0.3$ & $49.2 \pm 0.2$ & $47.7 \pm 0.9$ & $47.6 \pm 0.8$ & $44.7 \pm 0.03$ & $38.1 \pm 0.2$ \\
\hline
\end{tabular}

${ }^{a}$ Results are given for the 256-DPPC simulation $(T=309 \mathrm{~K})$ and for the 200-DPPC simulation $(T=323 \mathrm{~K})$. Values for the initial dimensions and volume are given but cannot be compared directly to the sterol-containing simulations. ${ }^{b}$ The initial volume of the simulation cell for the sterol-containing membranes was $241920 \AA^{3}$.

studies. ${ }^{71}$ Ergosterol and lanosterol were constructed from the cholesterol molecule using the software package Insight II. ${ }^{72}$

Since there are no experimental measurements for the surface area per DPPC in a binary sterol/DPPC system, the sterolcontaining membrane was constructed here in order to produce an initial mean value of the area per lipid of $50 \AA^{2}$, which is the average value from two previous cholesterol-DPPC MD simulations ${ }^{44,35}\left(45.6 \AA^{2}\right.$ and $\left.54.2 \AA^{2}\right)$. Thus, the initial dimensions of the primary cell chosen for the system were $x=$ $56.0 \AA, y=72.0 \AA, z=60.0 \AA$ using an orthorhombic box $(\alpha$ $=\beta=\gamma=90^{\circ}$ ). The center of the unit cell was set to the origin of the coordinate system, and the $z$-axis was chosen to coincide with the membrane normal. The lipid bilayer was surrounded by 1600 TIP3P ${ }^{73}$ water molecules leading to a 20 wt $\%$ hydration.

The NPT ensemble was chosen because a flexible simulation cell allows the lipid bilayer to expand or contract when sterol molecules are added so that the bilayer will adapt to its preferred state. Each system was allowed to relax into its preferred surface area per lipid, with all three simulation box coordinates adjusting independently.

Simulation Protocol. Initially, a 50-lipid system consisting of 30 DPPC, 20 cholesterol, and 400 water molecules was constructed. This system was energy-minimized for 1000 steps with the CG method, followed by 30000 steps of the ABNR minimization. Next, the system was heated to 309 or $323 \mathrm{~K}$ in $5-\mathrm{K}$ steps every 5000 timesteps. The system was equilibrated for $600 \mathrm{ps}$ at constant pressure (1 atm) and temperature (309 or $323 \mathrm{~K})$ using the extended-system Hoover algorithm with periodic boundary conditions as implemented in CHARMM. ${ }^{64}$ After a 600-ps equilibration, a 200-lipid system was created by duplicating and translating the original slab of the preequilibrated 50 lipids in the $x$ and $y$ directions. To construct the ergosterol/lanosterol membrane systems, the cholesterol molecules were replaced accordingly, and energy minimization and heating to 309 or $323 \mathrm{~K}$ was applied. Therefore, any differences arising in the structure and dynamics of the three sterol-containing membranes are due to the differences in their molecular structures and do not arise from differences in their initial configurations.

Before production dynamics, all the systems were equilibrated for $15 \mathrm{~ns}$. Long equilibration times are required for relaxing the molecules from their initial model-built configurations and for convergence of properties of these systems. ${ }^{52,74}$ The equilibration was checked by ensuring that the energy and volume distributions have a Gaussian shape. The time series of the total energy for each simulated system can be seen in Figure S1 (Supporting Information).

A separate simulation of the isolated sterols in vacuum at $T$ $=309$ and $323 \mathrm{~K}$ was also performed to assess their vacuum conformational flexibility.

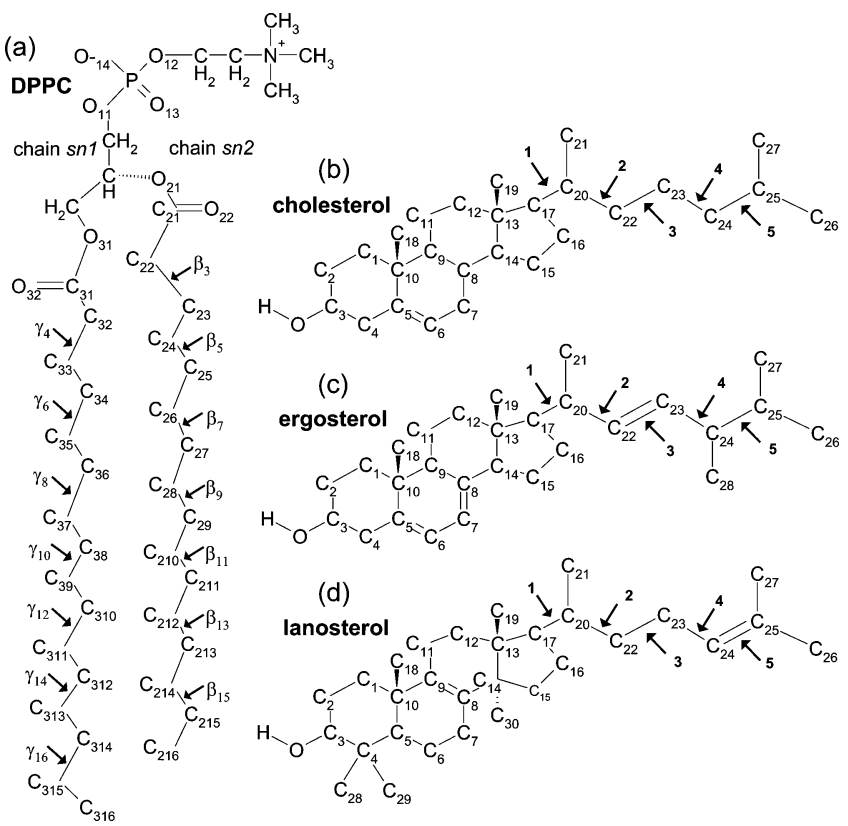

Figure 1. Chemical structure, atom numbering, and dihedral angle schemes of (a) DPPC, (b) cholesterol, (c) ergosterol, and (d) lanosterol.

Stability of the Simulations. The systems were allowed to adjust not only their volumes (density) but also their area-tothickness ratios. For the sterol-DPPC systems, the dimensions of the unit cell and the total volume of the systems do not show a drift in time, indicating stable simulations (See Figures S2 and S3 of the Supporting Information). The time series of the total energy for each system can be seen in Figure S1 (Supporting Information). The average cell dimensions $x, y, z$ and the unit cell volumes for all systems are listed in Table 1.

\section{Results and Discussion}

In what follows, the effect of each sterol on the membrane properties is examined at atomic detail. First, the ordering effect on the lipids, lipid packing, and the gauche populations on the DPPC acyl chains are discussed. The location of each sterol and its preferred orientation in the membrane is examined via electron density profiles and sterol tilt angles, respectively. The solvation of different groups in the sterol/DPPC systems is also studied. The chemical origins of the different membrane behavior upon addition of each sterol are discussed with respect to the sterol chemical structures.

Ordering of DPPC Acyl Chains. Many experimental and computational studies have been devoted to studying the effect of cholesterol on the DPPC acyl chain ordering. Here, we reexamine this and compare with ergosterol and lanosterol. A useful quantity for characterizing the order of the hydrocarbon 


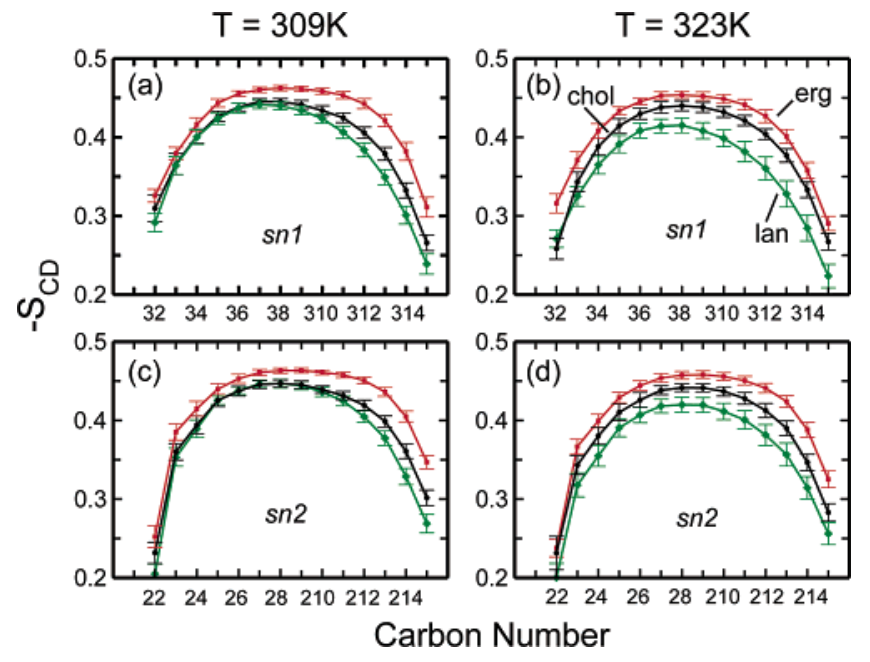

Figure 2. Order parameter profile for the DPPC in sterol-containing membranes. Cholesterol-DPPC, black line and circles; ergosterolDPPC, red line and squares; lanosterol-DPPC, green line and diamonds. (a) $T=309 \mathrm{~K}$, sn1 chain; (b) $T=323 \mathrm{~K}$, sn1 chain; (c) $T$ $=309 \mathrm{~K}$, sn 2 chain; and (d) $T=323 \mathrm{~K}$, sn 2 chain. The error bars represent standard deviations calculated by dividing the 8-ns trajectories into 100-ps pieces and calculating order parameter profiles for each.

chains in lipid bilayers is the deuterium NMR order parameter, $S_{\mathrm{CD}}$. An order parameter may be defined for every $\mathrm{CH}_{2}$ group in the DPPC hydrocarbon chains as follows,

$$
S_{\mathrm{CD}}^{i}=\frac{1}{2}\left(3\left\langle\cos ^{2} \theta_{\mathrm{CD}}\right\rangle-1\right)
$$

where $\theta_{\mathrm{CD}}$ is the angle between a CD-bond in NMR experiments ( $\mathrm{D}$ is deuterium in the experiment) or a $\mathrm{CH}$-bond (in the simulation) and the membrane normal (z-axis), and the superscript $i$ is the number of the carbon atom in the lipid acyl chain. The brackets indicate ensemble averaging over the two bonds in each $\mathrm{CH}_{2}$ group, the phospholipid molecules, and time. $S_{\mathrm{CD}}^{i}$ thus relates the geometry of the acyl chains to the orientational order parameter of the $\mathrm{C}-\mathrm{C}$ bond. The order parameter profiles of the acyl lipid chains for the present simulations of the three sterol/DPPC systems are given in Figure 2.

Figure 2 shows that the effect on the ordering of the acyl chains is significantly different for the three sterols, especially toward the bilayer center. At both temperatures, ergosterol induces the highest order in the bilayer, and lanosterol has the smallest ordering effect. The temperature increase lowers significantly the order parameters of the lanosterol-DPPC membrane, whereas there is just a slight decrease on the order parameters of the ergosterol- and cholesterol-DPPC membranes.

The changes in the order parameter profiles are different for the two lipid chains and depend on the position of the carbon atoms. Upon addition of the sterols, the $-S_{\mathrm{CD}}$ profile is roughly constant along DPPC carbons $\mathrm{C}_{6}$ to $\mathrm{C}_{8-11}$ (see Figure 2), particularly for the ergosterol-DPPC membrane, which takes values between 0.458 and 0.462 through carbons $C_{37}$ to $C_{310}$ for $T=309 \mathrm{~K}$ and the DPPC sn 1 chain. Lanosterol does not exhibit as constant values for the middle-carbon $-S_{\mathrm{CD}}$.

As can be seen in Figure 3, the presence of sterol results in large increases relative to the pure DPPC bilayer in the $-S_{\mathrm{CD}}$ profile of the hydrocarbon tails. ${ }^{75,76}$ These increases are likely to arise from the rigid steroid body of the sterols associating with the saturated hydrocarbon tails of DPPC. ${ }^{17,55}$

The addition of $50 \mathrm{~mol} \%$ cholesterol increases the values of $-S_{\mathrm{CD}}$ for a DPPC molecule by a factor of $\sim 2$ relative to a pure

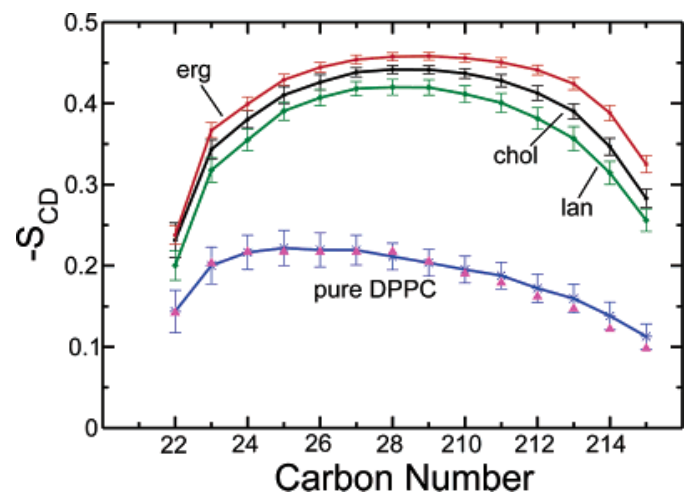

Figure 3. Order parameter profile for $T=323 \mathrm{~K}$ and the sn2 DPPC chain. Cholesterol-DPPC, black line; ergosterol-DPPC, red line; lanosterol-DPPC, green line; pure 72-DPPC membrane calculated from the present simulation in blue line and stars and NMR experimental results of ref 75 in magenta triangles. The error bars represent standard deviations calculated by dividing the 8-ns trajectories into 100ps pieces and calculating order parameter profiles for each.

membrane. ${ }^{44,75-78}$ The present simulation data for the pure DPPC sn2 chain are in excellent agreement with the corresponding NMR data in ref 75 (see Figure 3). The order parameter profile for cholesterol is also consistent with all previous simulation results obtained under similar conditions. ${ }^{35,44,55}$ The results obtained here for cholesterol, ergosterol and lanosterol (Figure 2) are also in good overall agreement with those obtained in an NMR study ${ }^{77}$ in which a similar ordering trend for cholesterol, ergosterol, and lanosterol on DMPC bilayers was observed. Furthermore, other NMR studies of lanosterol and cholesterol in a POPC bilayer ${ }^{79}$ indicated that lanosterol orders the DPPC acyl chains but does not have such a big effect as cholesterol, again in agreement with our simulations. Moreover, two additional NMR studies ${ }^{19,33}$ of cholesterol/ergosterol-DPPC bilayers concur with the present studies because they indicate that the chain ordering in ergosterol-containing membranes is stronger than in cholesterolcontaining membranes. This stronger lipid chain ordering could arise from the fact that the ergosterol structure inhibits DPPC chain conformational freedom even more than does cholesterol (see later in text).

Gauche Populations of DPPC Acyl Chains. An additional important conformational property of phospholipid chains is the fraction of gauche dihedral angles in the acyl tails. The transgauche isomerization is one of the fastest anharmonic motions experienced by the phospholipids (picosecond time scale), and it contributes to conformational disorder. Therefore, the gauche profiles can be also viewed as a measure of the order and organization in the bilayer system, which will affect other properties of the system. Here, the gauche fraction was determined by integrating each normalized torsion angle distribution for every DPPC hydrocarbon tail from -120 to $120^{\circ}$. The resulting values are plotted in Figure 4.

At $T=309 \mathrm{~K}$, the addition of any sterol to the gel DPPC system increases the gauche fraction, thus disordering the gel phase. In contrast, the presence of any of the three sterols to liquid DPPC $(T=323 \mathrm{~K})$ clearly decreases the total number of gauche defects per DPPC molecule at all carbon positions, thus ordering the liquid phase. The increase in the trans percentage is particularly evident in the positions $\beta 6-\beta 12$ and $\gamma 6-\gamma 12$, where the rigid steroid nucleus is located. (The notation for the dihedral angles in the lipid tails follows that adopted in ref 80 , which is commonly used.) The effect is smaller at the terminus, where the methyl group has more freedom of movement, and 


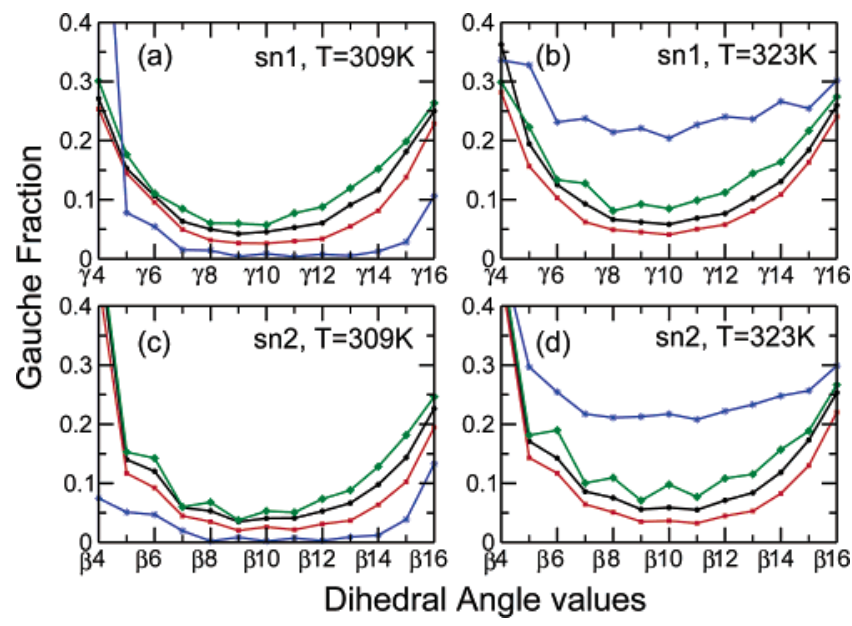

Figure 4. Positional dependence of gauche bond fraction in the DPPC acyl tail for the pure and sterol-containing systems. Pure DPPC, blue line with stars; cholesterol-DPPC, black line with circles; ergosterolDPPC, red line with squares; and lanosterol-DPPC, green line with diamonds. (a) $T=309 \mathrm{~K}$, sn 1 chain (angles $\gamma 4-\gamma 16$ ); (b) $T=323 \mathrm{~K}$, sn 1 chain (angles $\gamma 4-\gamma 16$ ); (c) $T=309 \mathrm{~K}$, sn 2 chain (angles $\beta 4-$ $\beta 16$ ); and (d) $T=323 \mathrm{~K}$, sn 2 chain (angles $\beta 4-\beta 16$ ). The notation for the dihedral angles in the lipid tails follows the notation adopted in ref 80 , which is commonly used (shown also in Figure 1). The lines are guides to the eye.

thus, the last phospholipid dihedral angle exhibits a significant number of gauche defects (3-fold symmetry). For the sn 2 chain, the gauche fractions of $\beta 4$ torsions remain unchanged at $T=$ $323 \mathrm{~K}$ and are close to those of liquid pure DPPC.

Infrared spectroscopic determination of the gauche fraction of gel/liquid and cholesterol-containing DPPC membranes ${ }^{81}$ indicates that the gel phase of DPPC is characterized by high conformational order, with gauche percentage values of $\approx 2 \%$ for $\gamma 7$ and $\gamma 11$, similar to the percentages of $1.5 \%$ for $\gamma 7$ and $1 \%$ for $\gamma 11$ calculated from the present simulation data. Other infrared spectroscopic studies ${ }^{82-84}$ have shown that at $323 \mathrm{~K}$, the liquid DPPC bilayer has gauche fractions of $\gamma 5,7,11,14$, 15,16 that are $0.21,0.30,0.20,0.17,0.40$, and 0.40 , respectively. Addition of $33 \mathrm{~mol} \%$ cholesterol reduced the corresponding values to $0.04,0.04,0.13$, and 0.11 by restricting the rotamer formation; no values were determined for positions 14 and 15 . These values are again in general agreement with the present calculated gauche fraction. The "odd-even" effect" for the gauche populations with $T>T_{\mathrm{m}}$ and with respect to the carbon position, that is, $P_{\gamma 6}<P_{\gamma 7}, P_{\gamma 8}<P_{\gamma 9}, P_{\gamma 10}<P_{\gamma 11}$, etc. ( $P$ : gauche population) is also observed (see Figure $4 \mathrm{~b}$ ).

Gauche populations of the DPPC acyl chains for ergosterolor lanosterol-containing membranes have not yet been experimentally determined. Our simulation data show that the gauche concentration of the lipid tails follows the order: gel DPPC $<$ ergosterol-DPPC $<$ cholesterol-DPPC $<$ lanosterol-DPPC $<$ liquid DPPC. This suggests that the ergosterol-containing membrane features the most conformational order in DPPC acyl chains and lanosterol, the least among the three sterols studied.

An increase in the hydrocarbon chain order and decrease in the fraction of gauche dihedral angles is accompanied by the increase of the hydrocarbon chain lengths. The increase of the acyl chain length becomes less pronounced with the inclusion of lanosterol than with the inclusion of ergosterol or cholesterol. The hydrophobic thickness of each bilayer was calculated by taking the average distance $\mathrm{C}_{21}-\mathrm{C}_{21}$ between DPPC that are found in opposite monolayers. At $T=323 \mathrm{~K}$, the hydrophobic thickness is $38.6 \pm 0.2 \AA$ for lanosterol, $39.3 \pm 0.2 \AA$ for cholesterol, and $39.9 \pm 0.2 \AA$ for ergosterol. At $T=309 \mathrm{~K}$,
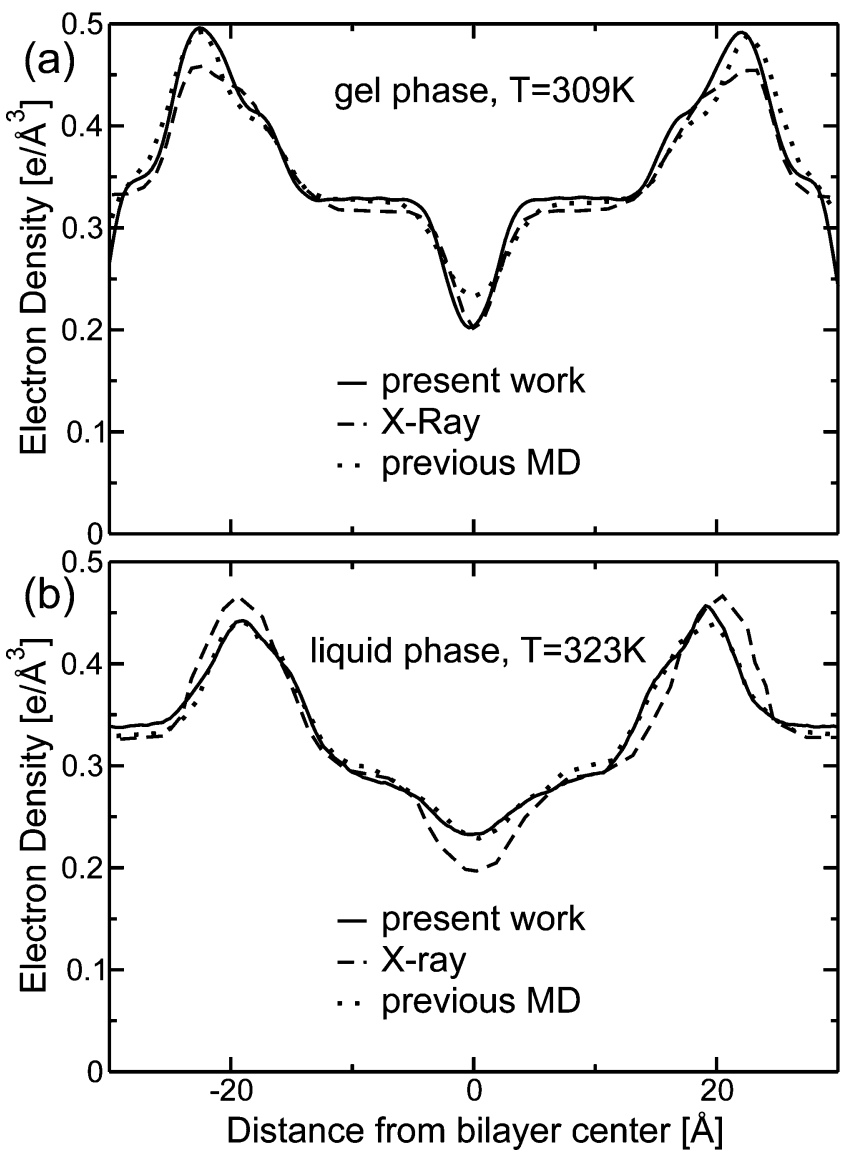

Figure 5. (a) Electron density profiles for the gel DPPC system at $T$ $=309 \mathrm{~K}$. The present simulation profile is the solid line, the experimental profile is taken from ref 85 , and the profile, from a previous simulation from ref 66. (b) Electron density profiles for the liquid DPPC system at $T=323 \mathrm{~K}$. The present simulation profile is again the solid line. The experimental profile is taken from ref 86 and the profile, from a previous simulation from ref 87 . The electron density profiles were calculated from the trajectories by dividing the simulation cells into $0.3-\AA$ slabs and determining the time-averaged number of electrons per slab using the coordinates of the atoms from the trajectories and assigning the corresponding number of electrons to the atomic centers.

the corresponding values are $38.8 \pm 0.4 \AA$ for lanosterol, 39.9 $\pm 0.2 \AA$ for cholesterol, and $40.4 \pm 0.3 \AA$ for ergosterol. Thus, we expect that the membrane thickness is smaller for lanosterol and larger for ergosterol, following the order of the gauche fractions. To evaluate the membrane thicknesses and compare it to available experimental data, we have calculated the electron density profiles for all simulated systems.

Electron Density Profiles. Information on the average structure of the lipid bilayer/water interface can be obtained from the electron density distributions of different types of atoms along the bilayer normal ( $z$-axis). These profiles also provide information on the thickness and organization of the molecules across the bilayer. Electron density profiles have been derived previously from X-ray diffraction of pure DPPC systems ${ }^{67}$ and from simulation studies for the cholesterol and ergosterol systems. ${ }^{55}$

In Figure 5a, electron density profiles for the pure gel DPPC system from low-angle X-ray diffraction ${ }^{85}$ and a previous MD simulation $^{66}$ (which used the CHARMM22 parameter set) are compared to the present simulation results. The distance between the maximums (in the present simulation, $44.7 \pm 0.03 \AA$ ) agrees very well with the experimental value of $45.0 \pm 1 \AA .{ }^{85}$ The flat methylene region and the terminal methyl trough are also 
well-reproduced. However, the shoulder appearing at the headgroup/glycerol region is less pronounced than in the X-ray data. In both the present simulation and the simulation of ref 66, the two high electron-density peaks show higher electron density in the headgroup region than that derived experimentally. One possible explanation might be that the head group motion is more restricted in the simulation than in the experiment. ${ }^{66}$ The electron density in the terminal methyl region is improved with respect to the $\mathrm{X}$-ray data in the present simulation relative to the previous simulation of ref 66 .

The electron density profile for the liquid DPPC system is given in Figure 5b, together with those derived from an X-ray diffraction experiment ${ }^{86}$ and a previous MD simulation with the CHARMM27 force field. ${ }^{87}$ The electron density profile calculated from the present trajectory is in excellent agreement with the simulation profile calculated in ref 87 . Both profiles are also in very good agreement with the $\mathrm{X}$-ray results. The distance between the two peaks is $38.1 \pm 0.2 \AA$, slightly smaller than the experimental distance of $39.8 \AA$. The electron density toward the middle of the bilayer is slightly higher in the simulation than in the experiment. One possible explanation for this difference might be that the terminal methyl groups are more mobile in the experiment than in the simulation, a fact also reflected in the gauche percentage of the DPPC terminal dihedral conformations $\left(0.40\right.$ in the experiment ${ }^{83}$ and 0.30 in the simulation).

In Figure 6, the simulation-derived electron density profiles for the sterol-containing membranes at $T=309$ and $323 \mathrm{~K}$ are shown together with the pure DPPC profile. The peaks are relatively sharp, allowing the thickness of the bilayer to be estimated from the peak-to-peak separation. The results are listed in Table 1. It is evident that all the sterols tend to "stretch" the bilayer, increasing its thickness relative to both the gel and the liquid DPPC phases, as has also been experimentally inferred from volumetric studies of cholesterol, ergosterol, and lanosterol in a DPPC bilayer. ${ }^{88}$ This effect is, however, more noticeable in the case of liquid DPPC, in which the sterol addition orders the membrane and as a result stretches the DPPC hydrocarbon tails (see also Ordering of DPPC Acyl Chains Section).

The membrane thickness of the gel-DPPC bilayer is close to that observed for the sterol-containing membranes. Addition of cholesterol, ergosterol, or lanosterol to gel-phase DPPC again increases the bilayer thickness (see Figure 6a). This effect was also observed in two neutron diffraction experiments..$^{71,89}$ The addition of these sterols to the pure lipid gel phase increases the gauche fraction of the phospholipid-chain dihedrals, decreasing the mean acyl chain ordering of the phospholipids ${ }^{9,11,19}$ (see also Gauche Populations of the DPPC Acyl Chains Section). With increasing DPPC acyl chain order, the membrane thickness increases as a result of the elongation of the lipid chains. ${ }^{90}$ Reduction of the acyl chain order in the gel phase would, thus, lead to reduction of the membrane thickness. However, upon incorporation of sterols into the gel phase, the opposite is observed: an increased membrane thickness. This increase was found to be not a consequence of increased lipid order, as in the liquid phase, but rather, of the decrease in the acyl chain tilt of the gel phase. Upon addition of the sterol, the gel-DPPC chain tilt (see Figure S4 of the Supporting Information) is lost, which leads to an increase in the bilayer thickness, even though the acyl chain order is reduced. Experimental results show an increase in membrane thickness of $\sim 3.5 \AA$ with the addition of 30 mol \% cholesterol to the gel phase of DMPC. ${ }^{71}$ This increase is consistent with the present simulation results within error, in which the gel phase membrane thickness increases $4.0 \pm 0.5$

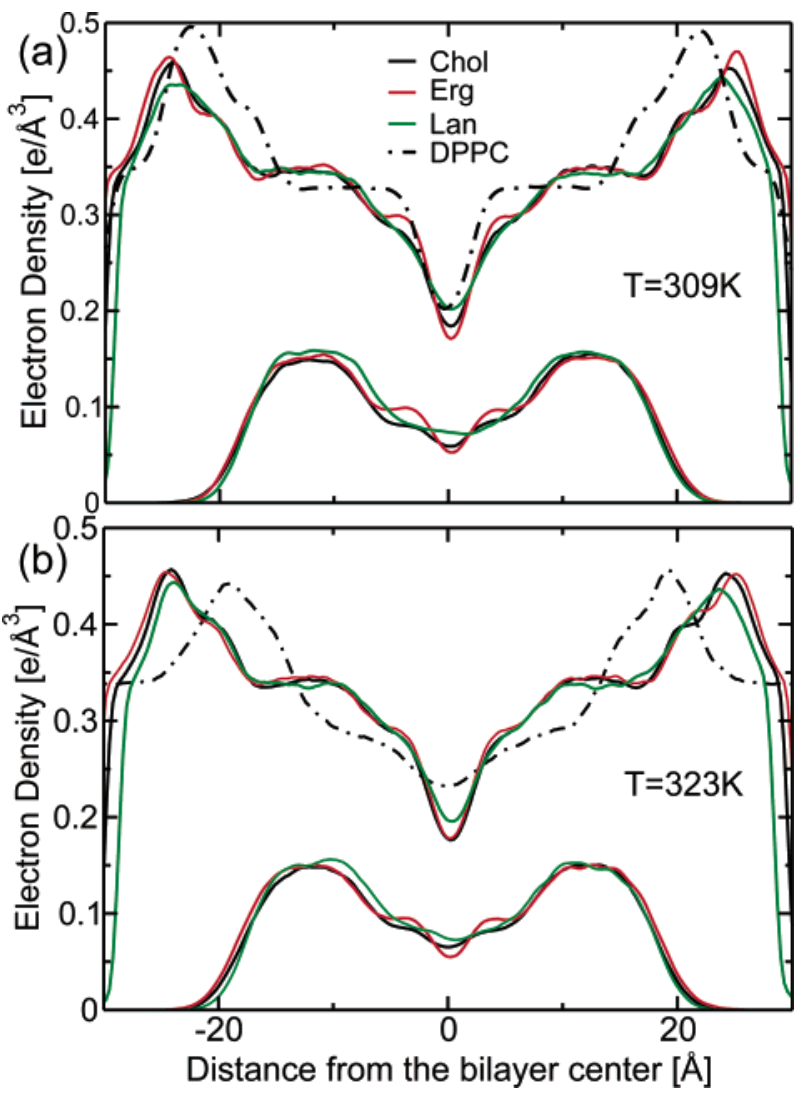

Figure 6. Electron density profiles for cholesterol-DPPC, black line; ergosterol-DPPC, red line; and lanosterol-DPPC, green line for (a) $309 \mathrm{~K}$ and (b) $323 \mathrm{~K}$ in comparison with the pure DPPC membrane (dashed-dotted black line). The upper curves in the figures represent the total electron density of the system, and the lower curves are the contributions of the three sterols. The electron density profiles were calculated from the trajectories by dividing the simulation cells into $0.3-\AA$ slabs and determining the time-averaged number of electrons per slab using the coordinates of the atoms from the trajectories and assigning the corresponding number of electrons to the atomic centers.

$\AA$ from $44.7 \AA$ in the gel phase to $48.7 \pm 0.5 \AA$ upon addition of $40 \%$ cholesterol.

Figure 6 and Table 1 show that only small differences are observed between the electron density profiles of the sterolcontaining membranes. In a recently performed small-angle, neutron-scattering study on cholesterol-, ergosterol-, and lanosterol-DMPC bilayers, it was also shown that only small differences occur in the influence of the sterols on membrane thickness. ${ }^{89}$ Specifically, for a DMPC bilayer at $303 \mathrm{~K}$ (liquid phase), the membrane thickness of an ergosterol-containing DMPC membrane (47 mol \% sterol) was $44.4 \AA$ and very close to the membrane thickness of the analogous cholesterolcontaining DMPC membrane (44.2 $\AA$ ). The lanosterol system had a slightly smaller thickness of $43.6 \AA$. The experimental membrane thickness, $h$, follows the order $h_{\text {lan }}<h_{\text {chol }}<h_{\text {erg. }}$. This behavior is also seen in the present MD simulations at both 309 and $323 \mathrm{~K}$, because the ergosterol membrane is thicker by $\approx 1 \AA$ than the cholesterol membrane and $\approx 2 \AA$ thicker than the lanosterol membrane (see Table 1). This result resembles that in an earlier MD study ${ }^{43}$ of cholesterol- and lanosterolDMPC bilayers at $50 \mathrm{~mol} \%$ sterol concentration and at $T=$ $308 \mathrm{~K}$, in which the authors report that the cholesterol membrane is $1 \AA$ thicker than that of lanosterol. In a recent MD study of cholesterol- and ergosterol-DMPC bilayers at $25 \mathrm{~mol} \%$ sterol concentration and $300 \mathrm{~K}$, the ergosterol-DMPC bilayer had a thickness of $41.5 \AA$, and the cholesterol-DMPC, $39.3 \AA .55$ However, the $25 \mathrm{~mol} \%$ cholesterol concentration is on the 


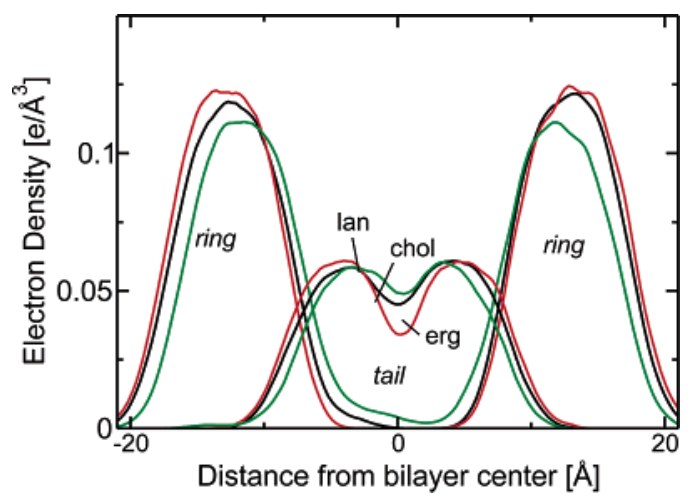

Figure 7. Electron density profiles for $T=323 \mathrm{~K}$ and for the common ring $\left(\mathrm{C}_{1}-\mathrm{C}_{19}\right)$ and tail $\left(\mathrm{C}_{20}-\mathrm{C}_{27}\right)$ atoms of the sterols. CholesterolDPPC, black line; ergosterol-DPPC, red line; and lanosterol-DPPC, green line. The central profiles are the electron density of the tail, and the outer profiles, the electron density of each sterol ring system.

border between the lo and the liquid phase,,${ }^{9,20}$ and a $25 \mathrm{~mol} \%$ ergosterol concentration is not in the lo phase (a concentration of over $30 \%$ is required), as experimental studies show. ${ }^{19}$

The contribution of each sterol to the total electron density of the membrane is shown in Figure 6. The electron density profiles arising from the sterols have two broad peaks. Because the ergosterol side chain has one methyl group more than the other two sterols, one should expect that its electron density near the bilayer center would be greater than the other two sterols; however, Figure 6 indicates that lanosterol exhibits at the center of the bilayer a slightly higher electron density, especially at $T=309 \mathrm{~K}$ (see Figure 6a, lower curve). Ergosterol, on the other hand, exhibits the lowest electron density in the bilayer midplane. Therefore, ergosterol is located closer to the water interface than the other two sterols.

To investigate further the $z$-positioning, the electron density profiles were calculated solely from the common ring and sidechain atoms of the three sterols, that is, $\mathrm{C}_{1}-\mathrm{C}_{19}$ of the ring atoms and $\mathrm{C}_{20}-\mathrm{C}_{27}$ of the sterol tail. In this manner, the positions of the three sterols in the bilayer can be directly compared. The results, shown in Figure 7, unambiguously demonstrate that lanosterol is found closer to the bilayer core than is cholesterol, by $2 \AA$ on average. The same conclusion was drawn in the MD study of ref 43, although for a DMPC bilayer and $11 \mathrm{~mol} \%$ sterol concentration. At $11 \mathrm{~mol} \%$ sterol concentration, the membrane is not in the liquid-ordered phase, and therefore, the membrane properties should be significantly different. Ergosterol is also slightly closer to the bilayer/water interface than is cholesterol and, thus, occupies the bilayer center the least.

Areas per Lipid in the Binary Systems. In the pure DPPC simulations, the average area per DPPC can be calculated by dividing the surface area of the simulation cell by the number of lipids per monolayer. However, in binary mixtures, there is no unique way of obtaining the area per lipid. The problem of calculating the correct area per DPPC in cholesterol-DPPC mixtures has been addressed in recent MD studies. ${ }^{35,50,53}$ The procedure used here is that of ref 35, in which the volume that a DPPC molecule occupies in a sterol-containing membrane in one frame of the trajectory is calculated as follows,

$$
V_{\mathrm{DPPC}}=\frac{V_{\mathrm{T}}-N_{\mathrm{W}} \times V_{\mathrm{W}}-N_{\mathrm{S}} \times V_{\mathrm{S}}}{N_{\mathrm{DPPC}}}
$$

where $V_{\mathrm{T}}$ is the total volume of the system, $N_{\mathrm{DPPC}}$ (here, 120) is the total number of the lipids, $N_{\mathrm{W}}$ (here, 1600) is the number of water molecules, $V_{\mathrm{W}}\left(29.24 \AA^{3}\right)$ is the volume of one water molecule at $T=309 \mathrm{~K}$ and $V_{\mathrm{W}}\left(29.56 \AA^{3}\right)$ is the volume of one water molecule at $T=323 \mathrm{~K}$ derived from the bulk water simulation, and $N_{\mathrm{S}}$ (here, 80) is the number of cholesterol molecules.

The volume of a cholesterol molecule, $V_{\mathrm{S}}=618.8 \AA^{3}$, was calculated as the average volume of one cholesterol molecule from the three published crystal structures. ${ }^{91-93}$ To obtain the volumes of ergosterol and lanosterol, the cholesterol volume was scaled with respect to the ergosterol/lanosterol van der Waals volumes, as calculated with CHARMM using standard van der Waals radii. The volumes thus obtained for ergosterol and lanosterol are 630.6 and $675.9 \AA^{3}$, respectively, that is, 2 and $8 \%$ larger than cholesterol. The increased volumes are due to the single methyl group on ergosterol and the three additional methyl groups in lanosterol. (In the MD study of ref 55, the ratio of van der Waals volumes for ergosterol/cholesterol was calculated to be 0.87 . Ergosterol has five fewer hydrogens than cholesterol but one additional methyl, and it is therefore expected to occupy a slightly larger volume than cholesterol.)

The area occupied by a DPPC molecule in a bilayer can be written as

$$
A_{\mathrm{DPPC}}(x)=\frac{2 V_{\mathrm{DPPC}}(x)}{h(x)}
$$

where $V_{\text {DPPC }}$ is the volume of the lipid and $h(x)$ is the average thickness of the membrane, which corresponds to the average distance between two phosphorus atoms in opposite layers.

At $T=323 \mathrm{~K}$ for the cholesterol-DPPC system, the volume per DPPC is $1194.4 \AA^{3}$, very close to the value reported by another MD simulation $\left(1189 \AA^{3}\right)^{35}$ for the same cholesterol concentration and temperature. For the same system from the present simulation data using eq 4, the area per lipid is estimated to be $49.6 \pm 0.3 \AA^{2}$ (see also Table 1 ), being smaller than the value of $54.2 \AA^{2}$ reported from MD simulation results in ref 35 , but very close to the value of $50.3 \AA^{2}$, reported again using MD simulations, by both refs 54 and 55 .

The area and volume per lipid for the ergosterol-DPPC and lanosterol-DPPC were also calculated, and the results are listed in Table 1. The time evolution of the area per lipid for each system is plotted in Figure 8 and does not show a drift, also confirming the stability of these simulations. Ergosterol induces the smallest area and volume per DPPC, and lanosterol, the largest. The area and volume per DPPC follow the order ergosterol $<$ cholesterol $<$ lanosterol for both temperatures studied. At the higher temperature, all values of the three areas per lipid are increased, but the above order is maintained. This effect was also observed in small-angle neutron scattering (SANS) data that measured thermal area expansion coefficients for the three different sterol-containing vesicles. ${ }^{89}$

The above results imply that ergosterol has the largest condensing effect on the membrane, and lanosterol, the lowest. Since all three sterols are rigid, they all have an ordering influence on liquid DPPC, as is also observed from the order parameter profiles of the DPPC acyl chains (see Ordering of the DPPC Acyl Chains Section). Therefore, acyl chain ordering not only reduces the number of gauche defects but also enhances the packing of the phospholipids, reducing cavity volumes between the molecules. All three sterols condense the bilayer (albeit to a different degree), decreasing the volume and area per DPPC molecule. Consequently, the total volume of the system is reduced relative to the pure liquid DPPC system, again in the order $V_{\text {erg }}<V_{\text {chol }}<V_{\text {lan }}$ (Table 1). 


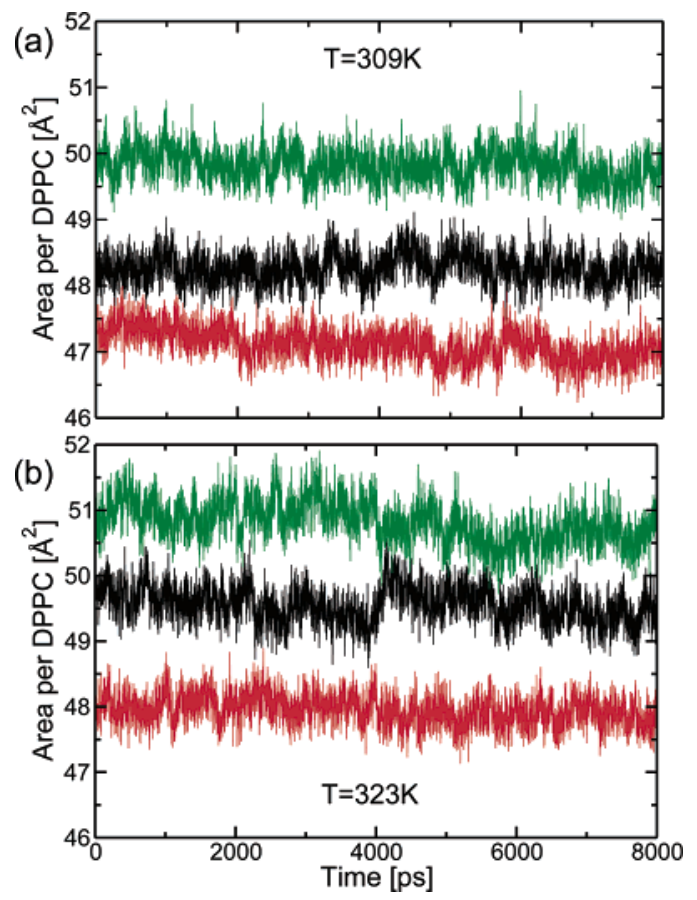

Figure 8. Time evolution of the area per DPPC in the sterol-containing membranes for (a) $T=309 \mathrm{~K}$ and (b) $T=323 \mathrm{~K}$. Cholesterol-DPPC, black; ergosterol-DPPC, red; lanosterol-DPPC, green.

At $T=309 \mathrm{~K}$, the area per lipid in a cholesterol-DPPC membrane is found to be $47.9 \pm 0.2 \AA^{2}$, that is, very close to the value of $49.3 \AA^{2}$ at $323 \mathrm{~K}$, thus indicating no significant change in the area per lipid with temperature. For a pure DPPC bilayer, the lipid phase transition occurs at $315 \mathrm{~K}$ and results in significantly different areas per lipid above and below the transition temperature. The area per lipid value in the sterolcontaining membrane is in the range of the experimentally measured values for the DPPC-gel phase at $309 \mathrm{~K}(47.9-52.3$ $\left.\AA^{2}\right),{ }^{67}$ although at $309 \mathrm{~K}$ with $40 \mathrm{~mol} \%$ cholesterol concentration, the lipids are still disordered (there is no specific packing of the phospholipids as in the gel state). These areas are significantly lower than the area per lipid for a pure DPPC bilayer in the liquid-crystalline state. ${ }^{67,86}$

The differences in the amount of the area per lipid reduction among the different sterols arise from their individual chemical structures. Ergosterol is stiffer and more rigid than the other two sterols, having two double bonds more than cholesterol. The extra methyl group in the ergosterol tail at position $\mathrm{C}_{24}$ also restricts the rotational motion of the sterol side chain as a result of steric hindrance arising in the bilayer core. Because ergosterol is conformationally and dynamically more restricted than cholesterol, it is likely to be more effective in inducing order and condensation in the bilayer. In contrast, lanosterol is the bulkiest of all three sterols, with three more methyl groups than cholesterol, which are attached to $\mathrm{C}_{4}$ and $\mathrm{C}_{14}$ and protrude from its otherwise flat $\alpha$-surface. These three extra methyl groups are likely to disrupt close packing of lanosterol and DPPC, resulting in less effective condensation.

To test the above assumptions and to shed light on the intermolecular interactions that might govern the observed differences, in the following sections, we have calculated the sterol tilt angles, van der Waals interaction energies, and pair radial distribution functions of different groups in the sterol/ DPPC systems.

Sterol Tilt Angle. The distributions of the tilt angles of sterols in the bilayer with respect to the bilayer normal are shown in Figure 9. The ring tilt angle is defined here as the angle between

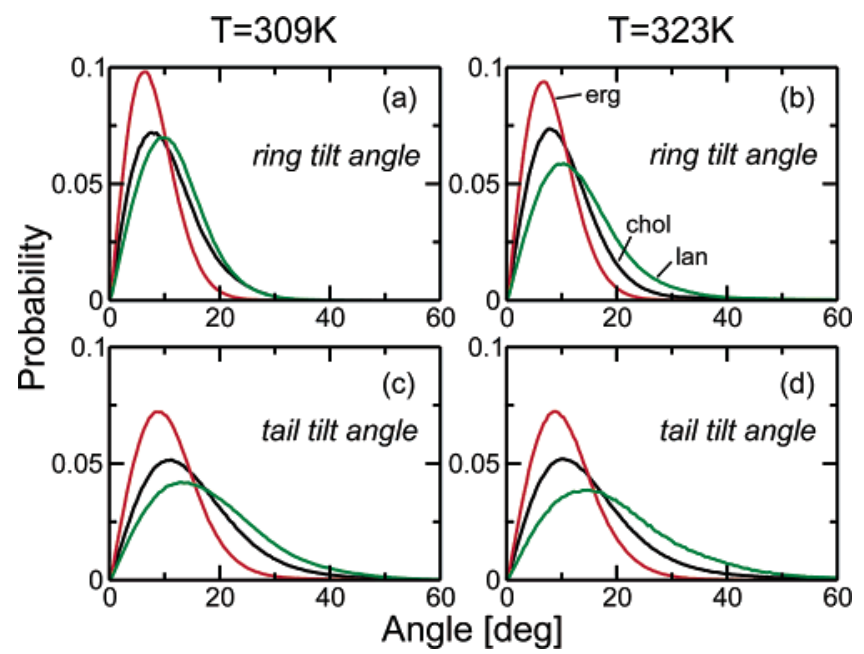

Figure 9. Tilt angle distributions between the bilayer normal and the vector connecting carbon atoms $\mathrm{C}_{3}$ and $\mathrm{C}_{17}$ in the sterol ring system for (a) $T=309 \mathrm{~K}$ and (b) $T=323 \mathrm{~K}$. Tilt angle between the bilayer normal and the vector connecting carbon atoms $\mathrm{C}_{17}$ and $\mathrm{C}_{25}$ for (c) $T$ $=309 \mathrm{~K}$ and (d) $T=323 \mathrm{~K}$. Cholesterol, black; ergosterol, red; and lanosterol, green.

the bilayer normal and the vector connecting carbon atoms $\mathrm{C}_{3}$ and $\mathrm{C}_{17}$ in the sterol ring system. Correspondingly, the tail tilt angle is defined as the angle between the bilayer normal and the vector connecting carbon atoms $\mathrm{C}_{17}$ and $\mathrm{C}_{25}\left(0^{\circ}\right.$ is parallel to the membrane normal)..$^{55}$

The mean values obtained for $T=323 \mathrm{~K}$ are $10.5,8.2$, and $13.6^{\circ}$ for the ring and $15.0,10.9$, and $19.9^{\circ}$ for the sterol tail for cholesterol, ergosterol, and lanosterol, respectively. Thus, for both the ring and tail tilt angles, cholesterol lies on average between ergosterol and lanosterol, with ergosterol the most closely aligned with the membrane normal and lanosterol, the least. With the increase of temperature, the sterol tilt angle probabilities do not change significantly.

Because ergosterol has an extra double bond in its steroid ring and a double bond in the middle of the sterol tail (between $\mathrm{C}_{22}$ and $\mathrm{C}_{23}$ ), it is stiffer than the other two sterols. This inherent stiffness causes the ergosterol molecule to be more aligned to the membrane normal than are the other two sterols. The extra methyl in the alkyl ergosterol tail does not significantly impair its ability to align with the DPPC hydrocarbon tails.

Although the lanosterol tail also contains a double bond, it is clearly the least aligned with the membrane normal. Because this double bond is located toward the end of the sterol tail and not in the middle, as in the case of ergosterol, it exhibits a smaller stiffening effect. Interestingly, the lanosterol tail is even more tilted than the saturated cholesterol tail. One possible explanation for this behavior is that lanosterol does not fit as well as ergosterol or cholesterol into the lipid bilayer (consistent with the observation that lanosterol induces the largest volume and the smallest order in the DPPC molecules). The poor fit of lanosterol is due to the rough $\alpha$-face of the molecule (lanosterol has three more methyls protruding from the $\alpha$-face than the other two sterols), which is evident in a schematic space-filling representation of the molecule (see Figure S5 of the Supporting Information).

The width of lanosterol's tilt angle distributions imply that it is more dynamic than the other sterols. As shown previously in the text (Area per Lipid Section), lanosterol creates the least densely packed membrane. Furthermore, it is located, on average, closer to the bilayer center than are the other two sterols. Since the bilayer center is the least densely packed region 

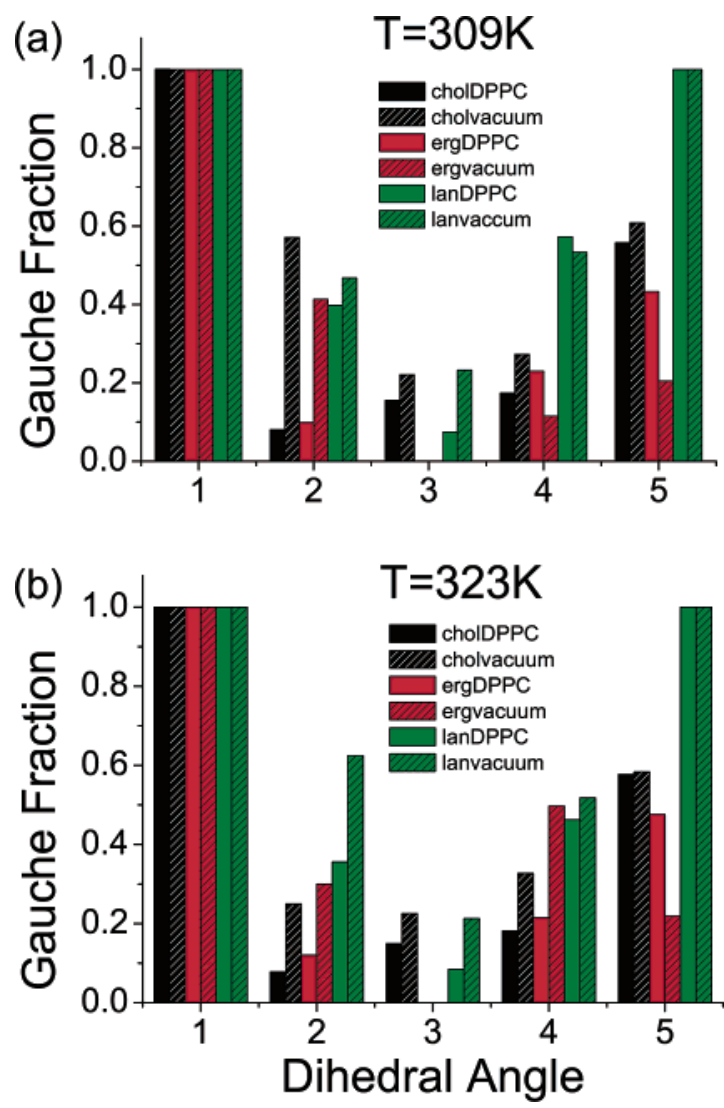

Figure 10. Positional dependence of gauche bond fraction in the sterol alkyl tail for (a) $T=309 \mathrm{~K}$ and (b) $T=323 \mathrm{~K}$. Cholesterol-DPPC, black; cholesterol in vacuum, shaded black; ergosterol-DPPC, red; ergosterol in vacuum, shaded red; lanosterol-DPPC, green; lanosterol in vacuum, shaded green. The dihedral angles $(1-5)$ are the sequential dihedral angles of the sterol tail: $1, \mathrm{C}_{16}-\mathrm{C}_{17}-\mathrm{C}_{20}-\mathrm{C}_{22} ; 2, \mathrm{C}_{17}-\mathrm{C}_{20}-$ $\mathrm{C}_{22}-\mathrm{C}_{23} ; 3, \mathrm{C}_{20}-\mathrm{C}_{22}-\mathrm{C}_{23}-\mathrm{C}_{24} ; 4, \mathrm{C}_{22}-\mathrm{C}_{23}-\mathrm{C}_{24}-\mathrm{C}_{25} ; 5, \mathrm{C}_{23}-\mathrm{C}_{24}-$ $\mathrm{C}_{25}-\mathrm{C}_{26}$ (see also Figure 1).

of the bilayer (see electron density profile, Figure 6), more space is available there. The pronounced increase in rotational disorder (i.e., gauche rotational isomers of the sterol tail) in lanosterol is energetically slightly unfavorable, but is favored on entropic grounds as a result of the available space in the bilayer midplane and in the loosely packed lanosterol-DPPC membrane.

The angle distributions are such that the more aligned a sterol is with the $z$-axis, the more order it induces in the DPPC acyl chains. Thus, ergosterol, which is more closely aligned with the membrane normal than the other sterols, induces more order in the DPPC hydrocarbon chains (as was also postulated in ref $55)$, as seen in Figure 2.

In Figure 10, the gauche fractions of the sterol tail dihedral angles in the DPPC membrane are plotted. The angles calculated are $1, \mathrm{C}_{16}-\mathrm{C}_{17}-\mathrm{C}_{20}-\mathrm{C}_{22} ; 2, \mathrm{C}_{17}-\mathrm{C}_{20}-\mathrm{C}_{22}-\mathrm{C}_{23} ; 3, \mathrm{C}_{20}-\mathrm{C}_{22}-$ $\mathrm{C}_{23}-\mathrm{C}_{24} ; 4, \mathrm{C}_{22}-\mathrm{C}_{23}-\mathrm{C}_{24}-\mathrm{C}_{25}$; and 5, $\mathrm{C}_{23}-\mathrm{C}_{24}-\mathrm{C}_{25}-\mathrm{C}_{26}$ (see Figure 1). To investigate any conformational restrictions arising from the membrane environment on the sterol tail dihedral angles, we also performed a vacuum simulation of the three sterols at $T=309$ and $323 \mathrm{~K}$.

For all three sterols, the tail dihedral 1 is always gauche, due to the steric interaction of the $\mathrm{C}_{21}$ methyl and the $\mathrm{C}_{19}$ methyl, which hinders the rotation around this dihedral both in the membrane and in vacuum. In the membrane environment, tail dihedrals 2 and 4 of cholesterol and ergosterol are constrained to be mostly in the trans conformation. In contrast, lanosterol has more freedom of movement in the tail and exhibits the gauche and trans conformations with almost equal probabilities for angles 2 and 4. The membrane environment restricts the rotation around dihedrals 2 and 4 relative to vacuum. Rotation around tail dihedral angle 3 is prohibited in the case of ergosterol due to the presence of the double bond. Although cholesterol and lanosterol have more freedom of movement, both show a preference for the trans conformation for dihedral 3, both in the membrane and vacuum environments. The terminal lanosterol dihedral angle is restricted to the trans conformation because of its terminal double bond. These results are also in agreement with an earlier conformational analysis of cholesterol and ergosterol in vacuum. ${ }^{94}$

The above results indicate that the difference in the location of the double bond in the sterol alkyl tail is important and results in conformational differences that affect the structural properties of the membrane.

Furthermore, the $\alpha$-surface of lanosterol is less planar, which may weaken van der Waals interactions between lipid hydrocarbon chains and lanosterol. ${ }^{22,95,96}$ To examine the rigidity of the steroid rings, the average values and standard deviations of some torsional angles of the steroid ring system were evaluated (see Table S1 of the Supporting Information). Apart from the expected differences in steroid ring torsional angles resulting from the difference in the position of the double bonds, no major differences among the three sterols are observed in the sterol ring. Temperature also has no effect.

Intermolecular Interactions. To further relate the differences in the chemical structure of the three sterols to their effects on the structure of the membrane, radial pair distribution functions, $g(r)$, for several types of interaction were calculated. The $g(r)$ were estimated here from the equation

$$
g(r)=\frac{V}{N}\left(\frac{n(r)}{4 \pi r^{2} \mathrm{~d} r}\right)
$$

where $V$ is the volume of the simulation box containing $N$ particles, and $n(r)$ is the number of particles $y$ in the sphere of radius $r$ and width $\mathrm{d} r$ around particle $x$. The first and second solvation shell occupancies were calculated from the respective $g(r)$ functions by integrating up to the first and second minima, respectively (see Table 2 ).

Hydration of the Sterol Hydroxyl. Water in the polar lipid region hydrates the lipid head groups. The radial distribution functions for the sterol hydroxyl hydrogen to water oxygen are plotted in Figure 11. The distributions for all three sterols exhibit a sharp first peak at $2 \AA$, corresponding to a first hydration shell around the hydroxyl group. The associated first hydration shell coordination numbers at $T=309 \mathrm{~K}$ are $1.6,1.5$, and 1.3 and at $T=323 \mathrm{~K}$ are $1.5,1.4$, and 1.3 for cholesterol, ergosterol, and lanosterol, respectively. Thus, the fact that cholesterol and ergosterol are located closer to the lipid/water interface than lanosterol means that they interact more often with water. Moreover, the two methyl groups attached to the $\mathrm{C}_{4}$ of lanosterol make its hydroxyl region more hydrophobic (see also Figure S5 of the Supporting Information), which may also contribute to lanosterol's being less hydrated.

Solvation of Sterols by DPPC Molecules in the Bilayer at $\boldsymbol{T}=\mathbf{3 2 3} \mathbf{K}$. The solvation of the sterol hydroxyl hydrogen by the carbonyl oxygen of both the sn1 and sn 2 chains is shown in Figure 12a and b. It has been reported from previous MD simulations that cholesterol is hydrogen-bonded with the DPPC carbonyl atoms. ${ }^{54,55}$ Indeed, integration of up to the first minimum of the $g(r)$ shows that here also, the sterol hydrogens are strongly solvated by the DPPC carbonyl oxygen. Cholesterol 
TABLE 2: First and Second Average Solvation Shells for Various Groups in the Simulation for $T=323 \mathrm{~K}$

\begin{tabular}{|c|c|c|c|c|c|c|}
\hline & \multicolumn{2}{|c|}{ DPPC-Chol } & \multicolumn{2}{|c|}{ DPPC-Erg } & \multicolumn{2}{|c|}{ DPPC-Lan } \\
\hline & first shell & second shell & first shell & second shell & first shell & second shell \\
\hline sterol hydroxyl $\mathrm{H}-\mathrm{O}$ water ${ }^{a}$ & 1.5 & 2.9 & 1.4 & 2.7 & 1.3 & 2.5 \\
\hline sterol hydroxyl $\mathrm{H}-\mathrm{O}_{22}$ & 5.0 & 11.2 & 6.0 & 13.2 & 4.0 & 9.2 \\
\hline sterol hydroxyl $\mathrm{H}-\mathrm{O}_{32}$ & 4.1 & 15.9 & 2.7 & 14.7 & 4.3 & 13.9 \\
\hline sterol $\mathrm{C}_{4}$-DPPC C $\mathrm{C}_{22}$ & 11.1 & 16.7 & 11.0 & 17.6 & 7.5 & 14.8 \\
\hline sterol $\mathrm{C}_{4}$-DPPC C $\mathrm{C}_{32}$ & 10.0 & 16.0 & 11.8 & 17.7 & 10.0 & 16.6 \\
\hline sterol ring C-DPPC $\mathrm{C}_{24}: \mathrm{C}_{210}$ & 7.3 & & 10.6 & & 10.1 & \\
\hline sterol ring C-DPPC $\mathrm{C}_{34}: \mathrm{C}_{310}$ & 6.2 & & 10.1 & & 8.9 & \\
\hline sterol tail C-DPPC C $\mathrm{C}_{216}$ & 9.2 & 17.7 & 8.5 & 16.9 & 10.0 & 19.1 \\
\hline sterol tail C-DPPC C $\mathrm{C}_{316}$ & 9.3 & 18.8 & 9.4 & 17.9 & 9.5 & 19.4 \\
\hline
\end{tabular}

${ }^{a}$ The first and second solvation shells were calculated by integrating the corresponding pair radial distribution function up to the first and second minima, respectively. $\mathrm{H}$, hydrogen atom; $\mathrm{C}$, carbon atom; $\mathrm{O}$, oxygen atom.

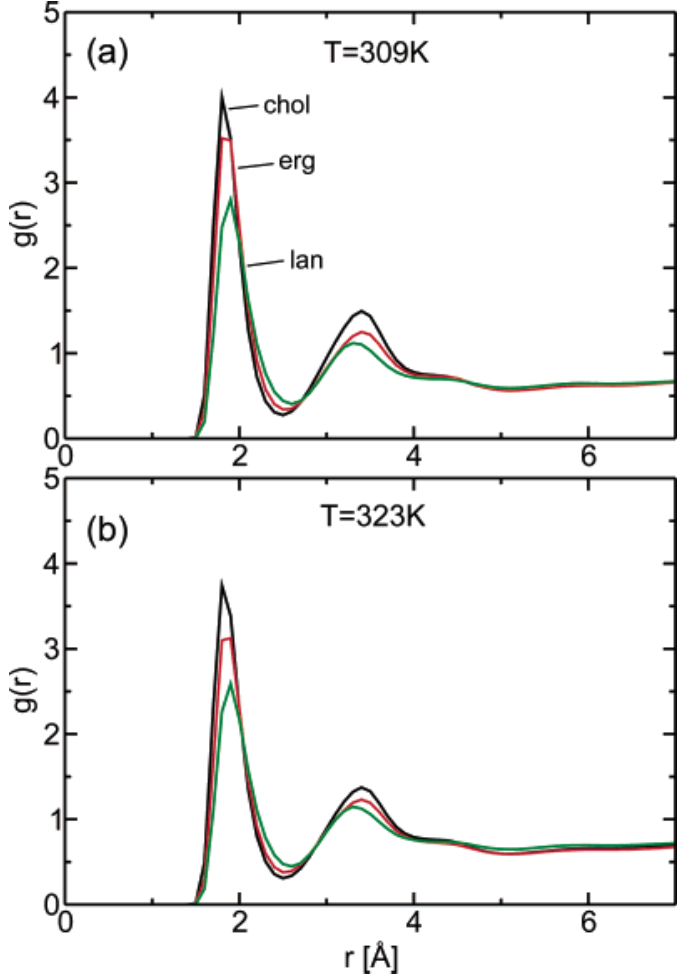

Figure 11. (a) Radial pair distribution functions of the sterol hydroxyl with water for (a) $T=309 \mathrm{~K}$ for cholesterol, black; ergosterol, red; and lanosterol, green; and (b) $T=323 \mathrm{~K}$ for cholesterol, black; ergosterol, red; and lanosterol, green.

and ergosterol show a clear preference for the sn2 carbonyl over the sn1 carbonyl, again consistent with previous observations. ${ }^{54,55}$ Lanosterol, on the other hand, does not show such a preference. The reason for these differences is, again, that cholesterol and ergosterol are located more toward the bilayer/ water interface, because the sn2 chain is closer to the bilayer/ water interface than sn1, and therefore, these two sterols are preferentially solvated. That ergosterol is the most solvated sterol by the $\mathrm{sn} 2$ carbonyl and the least solvated by the sn 1 carbonyl is again consistent with ergosterol's being the closest to the water interface.

The atom-packing around selected sterol ring atoms was also investigated. From the $g(r)$ functions plotted in Figure 12c, it is clear that the $\mathrm{C}_{4}$ of lanosterol interacts with the DPPC $\mathrm{C}_{22}$ or $\mathrm{C}_{32}$ carbons at a distance of $\approx 1 \AA$ further away than the corresponding interaction of the other two sterols. This observation can be attributed to the three extra methyls of lanosterol, that is, $\mathrm{C}_{28}, \mathrm{C}_{29}$, and $\mathrm{C}_{30}$, that protrude from its $\alpha$-face and prevent closer interaction of the steroid nucleus with the DPPC chain.
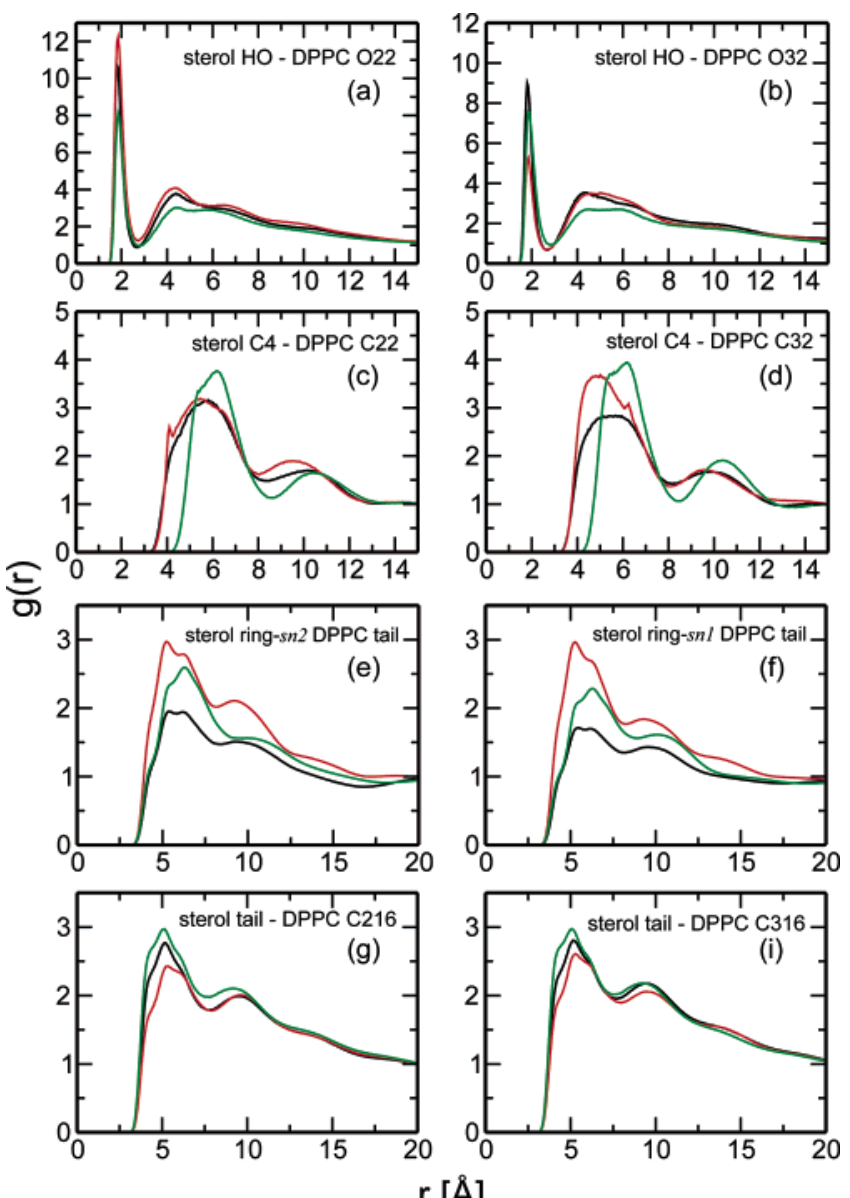

Figure 12. Radial pair distribution functions at $T=323 \mathrm{~K}$ of (a) sterol hydroxyl hydrogen relative to sn2 DPPC carbonyl oxygen $\mathrm{O}_{22}$, (b) sterol hydroxyl hydrogen relative to sn1 DPPC carbonyl oxygen $\mathrm{O}_{32}$, (c) sterol $\mathrm{C}_{4}$ relative to $\mathrm{sn} 2 \mathrm{DPPC} \mathrm{C}_{22}$, (d) sterol $\mathrm{C}_{4}$ relative to sn1 DPPC $\mathrm{C}_{32}$, (e) sterol ring carbon atoms $\mathrm{C}_{1}-\mathrm{C}_{19}$ relative to sn2 DPPC carbon atoms $\mathrm{C}_{24}-\mathrm{C}_{210}$, (f) sterol ring carbon atoms $\mathrm{C}_{1}-\mathrm{C}_{19}$ relative to sn1 DPPC carbon atoms $\mathrm{C}_{34}-\mathrm{C}_{210}$, (g) sterol side chain carbon atoms $\mathrm{C}_{20}-\mathrm{C}_{27}$ relative to sn2 DPPC terminal carbon atom $\mathrm{C}_{216}$, (i) sterol side chain carbon atoms $\mathrm{C}_{20}-\mathrm{C}_{27}$ relative to sn1 DPPC terminal carbon atom $\mathrm{C}_{316}$. Cholesterol, black; ergosterol, red; lanosterol, green.

The ergosterol steroid ring system $\left(\mathrm{C}_{1}-\mathrm{C}_{19}\right)$ has a higher probability of being solvated by both the $\mathrm{sn} 2$ and $\mathrm{sn} 1$ DPPC chains $\left(\mathrm{C}_{24}-\mathrm{C}_{210}\right.$ and $\left.\mathrm{C}_{34}-\mathrm{C}_{310}\right)$ than do the other two sterols, as shown from the respective $g(r)$ parts, e and $\mathrm{f}$, of Figure 12. Therefore, the ergosterol ring has, on average, more DPPC neighbors and induces a more densely packed membrane, in which cavities are reduced.

Finally, the atom-packing around the sterol tail was investigated. The $g(r)$ of the sterol side chain atoms $\mathrm{C}_{20}-\mathrm{C}_{27}$ with 
respect to the sn2 and sn1 DPPC terminal carbon atoms $\left(\mathrm{C}_{216}\right.$ and $\mathrm{C}_{316}$, respectively), are plotted in Figure $12 \mathrm{~g}$ and i. There is no preference in the solvation of the sterol tails for the sn1 or the sn2 DPPC acyl chains; however, it is evident that the lanosterol side chain is surrounded, on average, more by the terminal DPPC carbons, with $10.0 \mathrm{C}_{216}$ neighbors, as compared to 9.2 for cholesterol and 8.5 for ergosterol, again consistent with the fact that lanosterol is located more toward the bilayer midplane, as shown by the electron density profiles in Figure 7.

\section{Conclusions}

This article presents results from eight molecular dynamics simulations of DPPC membranes containing three different sterols: cholesterol, ergosterol, or lanosterol, at $40 \mathrm{~mol} \%$ concentration, that is, in the lo phase, and at temperatures of 309 and $323 \mathrm{~K}$. The simulations are compared with control simulations of the gel and liquid DPPC phases. The simulations were performed in order to study the differential effects of each sterol on the model membrane.

Several molecular dynamics studies have been previously performed on pure bilayers. ${ }^{68,97-105}$ These have generally been in good agreement with experiment, and the control simulations presented here, of DPPC in the gel phase at $309 \mathrm{~K}$ and liquid phase at $323 \mathrm{~K}$, are also in good agreement with the existing experimental data, including the electron density profiles, and in particularly good agreement with the available NMR order parameters of lipid acyl chains.

The results on sterol addition present a consistent picture of the effects of the sterols on the bilayer structure. At $323 \mathrm{~K}$, the addition of any of the three sterols to the membrane orders the DPPC acyl chains relative to the pure-DPPC liquid phase. The rigid sterol ring systems straighten the DPPC acyl chains, increase order parameters of the acyl chains, and inhibit transgauche conformational transitions. In contrast, at $T=309 \mathrm{~K}$, the sterols disorder the gel DPPC phase and increase the gauche populations of the DPPC acyl chain dihedrals. The pure gel phase lipids are close to optimally packed, and intercalation of flat ring systems cannot improve their packing. Consistent with the above picture, the area per lipid calculations indicate that the sterols condense the liquid membrane but expand the gel.

Temperature has a very small effect on the physical properties of the sterol/lipid systems between 309 and 323 K. However, although the chemical differences between the sterols are relatively small, they lead to significant differences in membrane structure. The ordering and condensing effect of ergosterol is found to be the highest, with lanosterol the lowest, and cholesterol in between. Important differences in membrane properties are schematized in Figure 13, in which the two extreme cases, ergosterol and lanosterol, are sketched.

The three extra methyls of lanosterol $\left(\mathrm{C}_{28}, \mathrm{C}_{29}, \mathrm{C}_{30}\right)$ with respect to the other two sterols, protrude from its otherwise flat $\alpha$-face and roughen its surface. In contrast, ergosterol has a smooth $\alpha$-face (as does cholesterol), one double bond more than lanosterol, and two more than cholesterol.

The result of the smoothness of ergosterol is that the ring packs closer to the lipid chains. This closer packing is seen in the relevant radial distribution functions (Figure 12c-f) but does not translate into more favorable interaction energies: for all three sterols, the lipid/sterol interaction energies are strongly negative (Figure S6 of the Supporting Information). The absence of a smooth $\alpha$-face holds lanosterol's steroid ring system further from the DPPC acyl chains than the other two sterols.

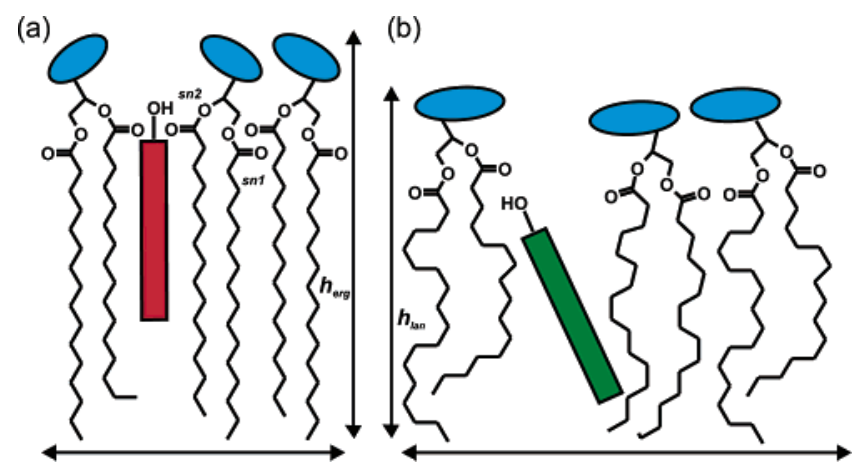

Figure 13. Schematic diagram depicting the following qualitative effects of (a) ergosterol and (b) lanosterol on the DPPC membrane. For the ergosterol system, the DPPC acyl chains are condensed (smaller area per lipid) and straighter (more trans conformations), and the membrane is thicker. Ergosterol is closer to the headgroup region, interacts more closely with the DPPC sn2 carbonyl than with the sn1 carbonyl, and is more closely aligned with the membrane normal. The behavior of cholesterol is intermediate between ergosterol and lanosterol.

One result of the closer packing of the lipid chains against the ergosterol ring is that the lipid chains become ordered, as reflected in increased order parameters (Figure 3). This order is also reflected in the percentage of the DPPC acyl chain dihedral angles in the gauche conformation, which again follows the order ergosterol < cholesterol < lanosterol, as does the area per DPPC. Thus, for ergosterol, the packing of the phospholipids is enhanced by reducing cavity volumes between the molecules. A consequence of the ordering of the DPPC acyl chains is a thickening of the membrane (increased headgroup/headgroup distance). In contrast, the bulkiest sterol (lanosterol) induces the least effective packing among the three sterols and the weakest ordering effect on the chains.

The two additional methyl groups at carbon $\mathrm{C}_{4}$ of lanosterol are very close to the hydroxyl group that is responsible for the amphipathic character of sterols. Their presence may weaken the ability of lanosterol to form hydrogen bonds to phospholipids and may play a role in another property seen here; namely, that the distance from the bilayer center follows the order ergosterol $>$ cholesterol $>$ lanosterol. Lanosterol is the most hydrophobic of the three molecules and, therefore, associates most with the hydrophobic bilayer core. Thus, lanosterol is located, on average, the closest to the bilayer center, and ergosterol, the closest to the bilayer/water interface, as schematically depicted in Figure 13. Among the consequences of this is the observation that although all three sterols do have a hydration shell around their hydroxyl groups (and at $T=309 \mathrm{~K}$ cholesterol is almost as hydrated as ergosterol), lanosterol is the least hydrated. The relative distance from the bilayer center also explains the closer interaction of the ergosterol and cholesterol hydroxyl groups with the sn 2 carbonyl group than with that of sn1 and that this preference is not seen for lanosterol.

The center of a lipid bilayer is relatively disordered and less well-packed. Thus, the observation that lanosterol is positioned closer to the center, together with the disordering effect of the rough $\alpha$-face, means that the lanosterol molecule has more room to move than do the other two sterols and, consequently, has a broader distribution of tilt angles relative to the membrane normal, with a significantly higher average tilt angle, which follows the order lanosterol $>$ cholesterol $>$ ergosterol.

The present simulation may shed light on the mechanism of the promotion of lipid rafts (domains) in eukaryotic plasma membranes. It has been suggested that lipid raft/domain formation is dependent on the sterol component having a 
structure that allows tight packing with lipids. ${ }^{21}$ Here, we have described in detail the result of this tighter sterol/lipid packing on the structure of a model DPPC membrane. The induction of lipid order, lipid/lipid packing condensation, sterol tilt angles, and sterol positioning relative to the membrane center may all play roles in raft formation. Fluorescence quenching and detergent-insolubility experiments have suggested that ergosterol is significantly more domain-promoting than cholesterol ${ }^{21}$ and that lanosterol is the weakest of the three, ${ }^{11,22}$ which is consistent with the present relative ordering effects.

Cholesterol adopts a behavior between the other two sterols: not as disordering as lanosterol but not as stiffening and ordering as ergosterol. The stronger ordering effect of cholesterol relative to lanosterol, revealed in detail in the present simulations, may well be part of the reason why the former has been evolutionarily preferred in the plasma membranes of higher vertebrates.

\section{Appendix}

Choice of the Optimal Surface Tension Value. The value of the surface tension, $\gamma$, that should be applied on a lipid bilayer in order to properly reproduce important bilayer properties, such as the area per lipid or the deuterium order parameters, is the subject of debate. ${ }^{101,106-112}$ It has been suggested that the surface tension must be zero for an unstressed bilayer at its free energy minimum (i.e., for a bilayer that is not subjected to osmotic stress). ${ }^{111}$ On the other hand, it has been pointed out that the microscopic and macroscopic implications of the change in the free energy of a bilayer with surface area are not the same. ${ }^{107}$ In refs 39 and 110, the CHARMM package was used with zero surface tension for simulating amphotericin B and cholesterol/ ergosterol and gramicidin $S$, respectively, in a membrane environment using the CHARMM force field. Therefore, the question remains of what should be the correct value of the applied surface tension in lipid bilayer simulations.

The area per lipid is generally considered to be a reliable criterion for validating and comparing calculations. Once the area per lipid is correct, most of the other properties of the bilayer appear to be reasonable, consistent with the observation that the large number of DPPC simulations available in the literature using different force fields and simulation methods result in bilayers with similar properties. ${ }^{60}$ It has been shown in previous simulations that the calculated surface area per lipid and the deuterium order parameters depend strongly on $\gamma .113$

Recently, a number of attempts have been made to simulate the liquid-crystalline state of a DPPC bilayer in CHARMM. ${ }^{101,109,113,114}$ In all of these studies, it is evident that the calculated area per lipid is sensitive to the used protocol and especially to the applied surface tension and the treatment of the electrostatics. ${ }^{115}$ It has also been argued that the required applied surface tension is dependent on the system size. ${ }^{100}$

In refs 101 and 113, exactly the same system of 72 DPPC molecules at $323 \mathrm{~K}$ was simulated with CHARMM. To achieve the observed experimental area per lipid of $62.9 \AA^{2}$, in ref 113 , it was estimated that the surface tension applied on a bilayer should be in the range $35-45 \mathrm{dyn} / \mathrm{cm}$, whereas a very different value of $\gamma=61 \mathrm{dyn} / \mathrm{cm}$ was required to produce an area per lipid of $62.2 \AA^{2}$ in ref 101 . One possible reason for this difference might be the fact that ref 113 used the PARM22b4b molecular mechanics force field parameter set, whereas ref 101 used CHARMM27. Different force fields might affect the surface tension required for a lipid bilayer. ${ }^{108}$

Recently, two additional CHARMM simulations on lipid bilayers using the $\mathrm{NP}_{\mathrm{n}} \gamma \mathrm{T}$ ensemble have been performed. In one simulation, an 80-DPPC bilayer patch was simulated, ${ }^{109}$
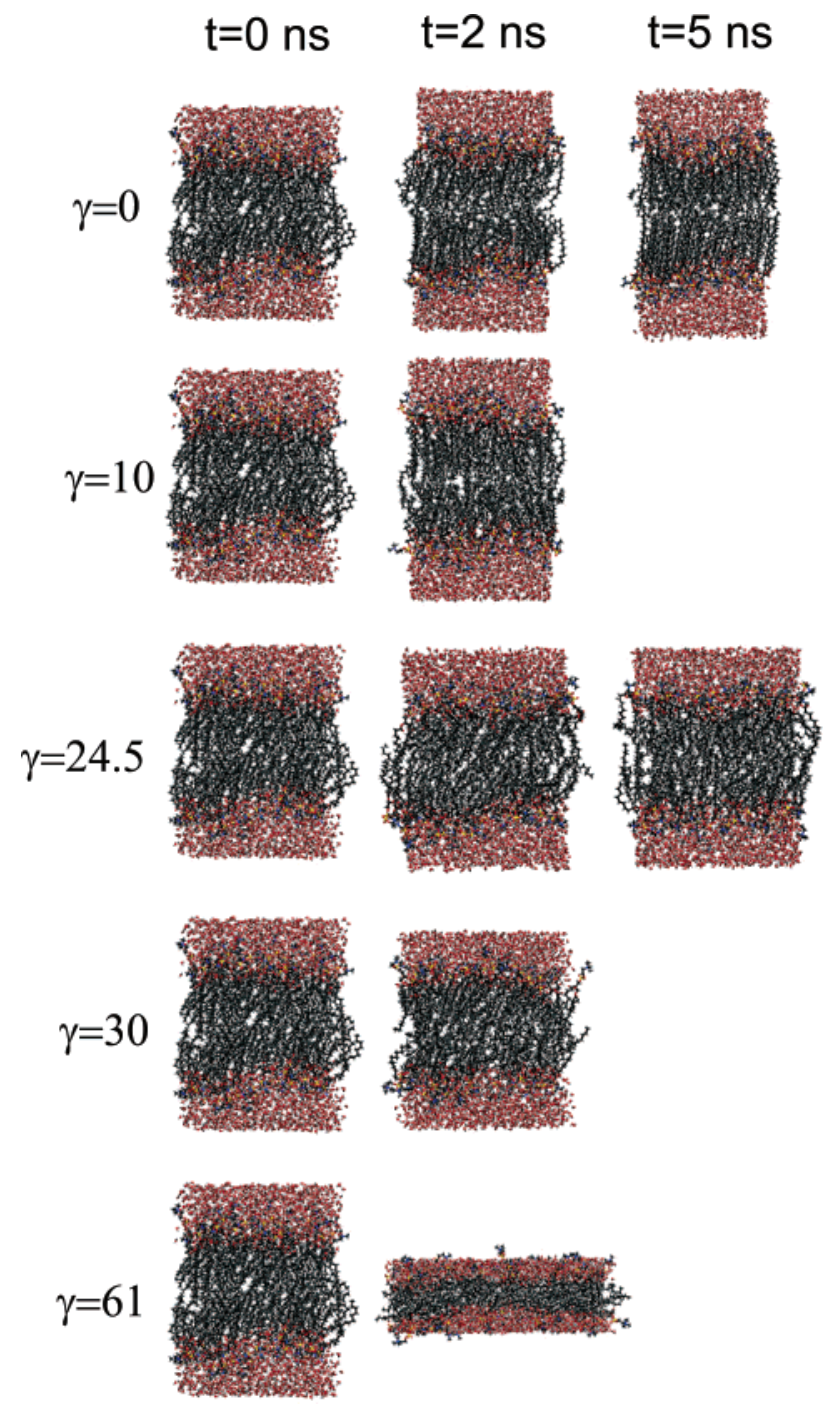

Figure 14. Snapshots of the liquid DPPC membrane (323 K) taken at $t=0,2$, and $5 \mathrm{~ns}$ (where available) simulated in the $\mathrm{NP}_{\mathrm{n}} \gamma \mathrm{T}$ ensemble with $\gamma=0,10,24.5,30$ and $61 \mathrm{dyn} / \mathrm{cm}$. Oxygen atoms are shown in red; carbon atoms, in black; nitrogen atoms, in blue; phosphorus atoms, in yellow; and hydrogen atoms, in gray. Pictures are in the $(z, x)$ plane.

requiring $17 \mathrm{dyn} / \mathrm{cm}$ to achieve an area per lipid of $67.0 \AA^{2}$. In the second simulation, a surface tension in the range of 25-30 $\mathrm{dyn} / \mathrm{cm}$ was required for a 90-DMPC bilayer patch. ${ }^{114}$

The effect of surface tension in lipid bilayers was also recently studied with the GROMACS ${ }^{116}$ force field. References 60,100 , and 115 used zero surface tension to simulate 128-DPPC bilayers. Reference 117 studied the effect of surface tension on the 128-DPPC bilayer and showed that to reproduce a surface area per lipid of $64 \AA^{2}$, a surface tension between 20 and 30 $\mathrm{dyn} / \mathrm{cm}$ was needed (depending on the system size).

To find the optimal value of $\gamma$ that reproduces the correct area per lipid for the fluid phase, here, a number of simulations were performed on the liquid (i.e., $T=323 \mathrm{~K}$ ) 72-DPPC bilayer with $\gamma=0,10,24.5,25,30$, and $61 \mathrm{dyn} / \mathrm{cm}$. In Figure 14, snapshots are shown, taken at $t=0,2$, and $5 \mathrm{~ns}$ (where available) of the liquid membrane with the different applied surface tensions.

Figures S7 and S8 (Supporting Information) show the variation of the area and volume per DPPC molecule obtained for different surface tensions as a function of time. It is evident from Figures 14 of the main text and S7a of the Supporting Information that the value of $\gamma=61 \mathrm{dyn} / \mathrm{cm}$ proposed by ref 
101 for a 72-DPPC bilayer over-suppresses the bilayer in the $z$-axis. Therefore, this simulation is not further analyzed.

At $\gamma=0$ dyn $/ \mathrm{cm}$, the area per lipid in the liquid phase is severely underestimated, and even at $t=5 \mathrm{~ns}$, it is clearly not converged (Figure S7a of the Supporting Information). Surface tension values of $\gamma=24.5$ and $\gamma=25 \mathrm{dyn} / \mathrm{cm}$ reasonably reproduce the experimental area per lipid. The mean values for the area per lipid observed in these simulations are $62.6 \pm 1.2$ and $63.7 \pm 0.8 \AA^{2}$. Here, we find that, using the CHARMM27 parameter set for the same 72-lipid system used in both simulations of refs 113 , a $\gamma$ value of $24.5 \mathrm{dyn} / \mathrm{cm}$ is required to reproduce an area per lipid of $62 \AA^{2}$, and $\gamma=25 \mathrm{dyn} / \mathrm{cm}$ is required for an area per lipid of $64 \AA^{2}$. Therefore, for the simulation of the pure bilayer at $T=323 \mathrm{~K}$, an applied surface tension of $24.5 \mathrm{dyn} / \mathrm{cm}$ was chosen, which best reproduces the experimental area per lipid ${ }^{86}\left(62.9 \pm 0.013 \AA^{2}\right)$.

Estimating the surface area per lipid in complex heterogeneous systems is difficult, and in these cases, the choice of $\gamma$ can be complicated. ${ }^{114}$ The addition of compounds to a pure phospholipid membrane is expected to influence the surface tension of the bilayer. From various simulation results, it has been suggested that the area per lipid in a cholesterol-DPPC bilayer is close to $50 \AA^{2} .^{44,35}$ This area per lipid is closer to that of a gel state $\left(47.9 \AA^{2}\right)^{67}$ than to that of a liquid state $(62.9$ $\left.\AA^{2}\right) .{ }^{67}$ Since the gel phase of a DPPC bilayer has been successfully simulated with $\gamma=0 \mathrm{dyn} / \mathrm{cm}$, we have chosen to also perform the sterol-DPPC simulations at $\gamma=0 \mathrm{dyn} / \mathrm{cm}$ in order to reduce equilibration times.

Area and Volume per Lipid vs Surface Tension in the Liquid DPPC Bilayer. The volume of a lipid molecule, $V_{\text {DPPC }}$, was calculated as

$$
V_{\mathrm{DPPC}}=\frac{V-N_{\mathrm{W}} V_{\mathrm{W}}}{N_{\mathrm{DPPC}}}
$$

where $V_{\mathrm{W}}$ is the volume of one water molecule, determined by a separate NPT simulation as described in the Methods Section. The volume of a water molecule was found to be $29.6 \pm 0.2$ $\AA^{3}$ for $323 \mathrm{~K}$ and $29.2 \pm 0.2 \AA^{3}$ for $309 \mathrm{~K}$.

The average DPPC volume is $1219.6 \pm 8.9 \AA^{3}$, within $2 \%$ of the experimental values reported by refs 118 and 97 . This value is remarkably stable, with a standard deviation of $<1 \%$. The volume per lipid does not exhibit any system-size dependence, being $1221.0 \pm 5.2 \AA^{3}$ for the 200-lipid system and $1219.6 \pm 8.9 \AA^{3}$ for the 72-lipid system.

The fluctuations in the area per lipid are considerably larger than the volume, and the equilibrium value exhibits a slight size dependence for the liquid DPPC membrane (see Figure S7b of the Supporting Information). The mean area per lipid for the 72-lipid system is $62.6 \AA^{2}$ and for the 200-lipid system is $64.6 \AA^{2}$ at $\gamma=24.5 \mathrm{dyn} / \mathrm{cm}$. These values are in close agreement with another MD study, ${ }^{100}$ in which a similar dependence of the area per lipid on the system size was also found (61.1 $\AA^{2}$ for a 64-lipid system and $63.0 \AA^{2}$ for a 256 lipid system at $T=323 \mathrm{~K}$ ).

Area and Volume per Lipid in the Gel DPPC Bilayer. For the gel DPPC simulation, studied at $\gamma=0 \mathrm{dyn} / \mathrm{cm}$, no major fluctuations in the area or the volume per lipid were observed, contrary to the case of the liquid DPPC bilayer area fluctuations. System size effects are also much smaller in this system than in the liquid DPPC simulation (see Figure S7b of the Supporting Information). The area per lipid is $47.3 \pm 0.5 \AA^{2}$ for the 64lipid system and $46.8 \pm 0.2 \AA^{2}$ for the 256-lipid system, both very close to the experimentally reported value of $47.2 \pm 0.5$
$\AA^{2} .{ }^{119}$ The simulation study of a gel-phase lipid bilayer carried out in ref 66, the simulation protocol of which was followed here, gave a somewhat smaller surface area per lipid of $45.4 \pm$ $0.5 \AA^{2}$

The volume per lipid in the gel phase, calculated from the present simulation, is $1110.4 \pm 3.0 \AA^{3}$ for the 256-lipid system and $1113.8 \pm 6.1 \AA^{3}$ for the smaller 64-lipid system. These two values are the same within the statistical error, and therefore, there is no system size effect for the gel phase DPPC bilayer. The time evolution of the volume per DPPC can be seen in Figure S8b (Supporting Information). This Figure shows that there is no drift in the time evolution of the volume per lipid and also that different system sizes produce the same volume per lipid. A depiction of the final frame of the gel systems is shown in Figure S4 of the Supporting Information. The characteristic tilt of the gel phase is seen in the $(x, z)$ plane for both the 64- and the 256-lipid simulations.

Time Evolution of the Unit Cell Dimensions with Different Surface Tensions. In Figure S2 of the Supporting Information, the time evolution of the $x, y$, and $z$ dimensions for the different systems is plotted. For the pure liquid DPPC systems simulated with $\gamma=0,10,30$, or $61 \mathrm{dyn} / \mathrm{cm}$ (see Figure $\mathrm{S} 2$ of the Supporting Information), the $x$ and $z$ dimensions drift in time, as does the area per lipid. Therefore, if the area per lipid drifts in time, other properties of the system may also not be converged. ${ }^{60}$ The observed drift in the area per lipid indicates that the simulated systems with the above-mentioned surface tensions are not yet equilibrated. However, for $\gamma=24.5$ and $\gamma$ $=25 \mathrm{dyn} / \mathrm{cm}$, the $x$ and $z$ dimensions and the area per lipid are stable, indicating that these systems are, indeed, equilibrated.

Acknowledgment. We thank A. L. Tournier and V. KurkalSiebert for fruitful discussions and R. M. Venable for supplying us the coordinates for the gel and the liquid pure DPPC bilayers. L. Nilsson is acknowledged for help with CHARMM; B. Costescu and S. Friedel, for system administration. We acknowledge funding from BMBF Grant 03SHE2HD and the Deutsche Forschungsgemeinschaft (DFG). Calculations were performed on the HELICS, IWR-Universität Heidelberg (HBFG funds, hww cooperation), and on the Computational Molecular Biophysics Group cluster.

Supporting Information Available: Supporting figures indicating the equilibration of the systems, space-filling models of the three sterol molecules, the final frames of the gel DPPC membrane simulation, interaction energy distributions, and a table with selected calculated torsional angles of the steroid rings are provided. This material is available free of charge via the Internet at http://pubs.acs.org.

\section{References and Notes}

(1) Alberts, B.; Bray, D.; Lewis, J.; Raff, M.; Roberts, K.; Watson, J. Molecular Biology of the Cell; Garland Publishing Inc.: New York and London, 1994.

(2) Finegold, L. Cholesterol in Membrane Models; CRC Press: Boca Barton, FL, 1985.

(3) Kusumi, A.; Tsuda, M.; Akino, T.; Ohnishi, O.; Terayama, Y. Biochemistry 1983, 22, 1165-1170.

(4) Bloch, K. In Biochemistry of Lipids and Membranes; Vance, J. E., Vance, D. E., Eds.; Benjamin/Cummins Publishing Co. Inc.: New York, 1985; pp 1-24.

(5) Simons, K.; Toomre, D. Nat. Rev. Mol. Cell Biol. 2000, 1, 31-39.

(6) Haines, T. H. Prog. Lipid Res. 2001, 40, 299-324.

(7) Risley, J. M. J. Chem. Ed. 2002, 79, 377-384.

(8) Polozov, I. V.; Gawrisch, K. Biophys. J. 2006, 90, 2051-2061.

(9) Vist, M. R.; Davis, J. H. Biochemistry 1990, 29, 451-464.

(10) Zuckermann, M. J.; Ipsen, J. H.; Mouritsen, O. G. In Cholesterol in Membrane Models; CRC Press: Boca Barton, FL, 1993; pp 223-256. 
(11) Miao, L.; Nielsen, M.; Thewalt, J.; Ipsen, J.; Bloom, M.; Zuckermann, M. J.; Mouritsen, O. G. Biophys. J. 2002, 82, 1429-1444.

(12) Mouritsen, J. H. I. O. G.; Zuckermann, M. J. Biophys. J. 1989, 56, $661-667$.

(13) Ipsen, J. H.; Karlstroem, G.; Mouritsen, O. G.; Wennerstroem, H.; Zuckermann, M. J. Biochim. Biophys. Acta 1987, 905, 162-172.

(14) McConell, H. M.; Radhakrishnan, A. Biochim. Biophys. Acta 2003 , $1610,159-173$

(15) Keller, S. L.; Pitcher, W. H.; Huestis, W. H.; McConnell, H. M. Phys. Rev. Lett. 1998, 81, 5019-5022.

(16) Radhakrishnan, A.; Anderson, T. G.; McConnell, H. M. Proc. Natl. Acad. Sci. U.S.A. 2000, 97, 12422-12427.

(17) Anderson, T. G.; McConnell, H. M. Biophys. J. 2001, 81, 27742785.

(18) Silvius, J. R. Biochim. Biophys. Acta 2003, 1610, 174-183.

(19) Hsueh, Y.-W.; Gilbert, K.; Trandum, C.; Zuckermann, M.; Thewalt, J. Biophys. J. 2005, 88, 1799-1808.

(20) Clarke, J. A.; Heron, A. J.; Seddon, J. M.; Law, R. V. Biophys. J. 2006, 90, 2383-2393.

(21) Xu, X.; Bittman, R.; Duportail, G.; Heissler, D.; Vilcheze, C.; London, E. J. Biol. Chem. 2001, 276, 33540-33546.

(22) Xu, X.; London, E. Biochemistry 2000, 39, 843-849.

(23) Bacia, K.; Schwille, P.; Kurzchalia, T. Proc. Natl. Acad. Sci. U.S.A.

2005, 102, 3272-3277.

(24) Fantini, J.; Garmy, N.; Mahfoud, R.; Yahi, N. Exp. Rev. Mol. Med.

2002, 1, 1-22.

(25) Baron, G. S.; Wehrly, K.; Dorward, D. W.; Chesebro, B.; Caughey, B. EMBO J. 2002, 21, 1031-1040.

(26) Tierney, K. J.; Bock, D. E.; Longo, M. L. Biophys. J. 2005, 89, $2481-2493$

(27) Arora, A.; Raghuraman, H.; Chattopadhyay, A. Biochim. Biophys. Acta 2004, 318, 920-926.

(28) Beattie, M. E.; Veatch, S. L.; Stottrup, B. L.; Keller, S. L. Biophys. J. 2005, 89, 1760-1768

(29) Soubias, O.; Jolibois, F.; Massou, S.; Milon, A.; Reat, V. Biophys. J. 2005, 89, 1120-1131.

(30) Orädd, G.; Lindblom, G.; Westerman, P. W. Biophys. J. 2002, 83 , 2702-2704

(31) Scheidt, A. H.; Huster, D.; Gawrisch, K. Biophys. J. 2005, 89 $2504-2512$

(32) Martinez, G. V.; Dykstra, E. M.; Lope-Piedrafita, S.; Brown, M. F. Langmuir 2004, 20, 1043-1046.

(33) Endress, E.; Bayerl, S.; Prechtel, K.; Maier, C.; Merkel, R.; Bayerl, T. M. Langmuir 2002, 18, 3292-3299.

(34) Doxastakis, M.; Sum, A. K.; de Pablo, J. J. J. Phys. Chem. B 2005, 109, 24173-24181.

(35) Hofsä $\beta$, C.; Lindahl, E.; Edholm, O. Biophys. J. 2003, 84, 21922206. 086.

(36) Filippov, A.; Orädd, G.; Lindblom, G. Biophys. J. 2003, 84, 3079-

(37) Almeida, P. F. F.; Vaz, W. L. C.; Thompson, T. E. Biochemistry 1992, 31, 6739-6747.

(38) Baudry, J.; Tajkhorshid, E.; Molnar, F.; Phillips, J.; Schulten, K. J. Phys. Chem. B 2001, 105, 905-918.

(39) Mihailescu, D.; Smith, J. C. Biophys. J. 2000, 79, 1718-1730.

(40) Berneche, S.; Nina, M.; Roux, B. Biophys. J. 1998, 75, 16031618.

(41) Forrest, L.; Kukol, A.; Arkin, I.; Tielman, A.; Sansom, M. Biophys. J. 2000, 78, 55-69.

(42) Flock, D.; Helms, V. Biophys. J. 2004, 87, 65-74.

(43) Smondyrev, A.; Berkowitz, M. L. Biophys. J. 2001, 80, 16491658.

(44) Smondyrev, A. M.; Berkowitz, M. L. Biophys. J. 1999, 77, 20752089.

(45) Chiu, S. W.; Jacobsson, E.; Scott, H. L. Biophys. J. 2001, 80, 1104-

(46) Tu, K.; Klein, M. L.; Tobias, D. J. Biophys. J. 1998, 75, 21472156.

(47) Edholm, O.; Nyberg, A. M. Biophys. J. 1996, 63, 1081-1089.

(48) Robinson, A. J.; Richards, W. G.; Thomas, P. J.; Hann, M. M. Biophys. J. 1995, 68, 164-170.

(49) Gabdoulline, R. R.; Vanderkooi, G.; Zheng, C. J. Phys. Chem. 1996, $100,15942-15946$

(50) Chiu, S. W.; Jacobsson, E.; Mashl, R. J.; Scott, H. L. Biophys. J. 2002, 83, 1842-1853.

(51) Pasenkiewicz-Gierula, M.; Rog, T.; Kitamura, K.; Kusumi, A. Biophys. J. 2000, 78, 1376-1389.

(52) Pitman, M. C.; Suits, F.; Mackerell, A. D., Jr.; Feller, S. E. Biochemistry 2004, 43, 15318-15328.

(53) Pandit, S. A.; Vasudevan, S.; Chiu, S. W.; Mashl, R. J.; Jacobsson, E.; Scott, H. L. Biophys. J. 2004, 87, 1092-1100.

(54) Pandit, S. A.; Bostick, D.; Berkowitz, M. L. Biophys. J. 2004, 86 , $1345-1356$
(55) Czub, J.; Baginski, M. Biophys. J. 2006, 90, 2368-2382

(56) Koynova, R.; Caffrey, M. Biochim. Biophys. Acta 1998, 1376, 91 145

(57) Brooks, B. R.; Bruccoleri, R.; Olafson, B. D.; States, D. J.; Swaminathan, S.; Karplus, M. J. Comp. Chem. 1983, 4, 187-217.

(58) Cournia, Z.; Smith, J. C.; Ullmann, G. M. J. Comp. Chem. 2005 $26,1383-1399$

(59) Darden, T.; York, D.; Pedersen, L. J. Chem. Phys. 1993, 98, 1008910092.

(60) Anézo, C.; de Vries, A. H.; Höltje, H.-D.; Tieleman, D. P.; Marrink,

S. J. J. Phys. Chem. B 2003, 107, 9424-9433.

(61) Verlet, L. Phys. Rev. 1967, 159, 98-103.

(62) Hill, T. L. An Introduction to Statistical Thermodynamics; Dover Publications Inc.: New York, 1986.

(63) Zhang, Y.; Feller, S. E.; Brooks, B. R.; Pastor, R. W. J. Chem. Phys. 1995, 103, 10252-10266.

(64) Feller, S. E.; Zhang, Y.; Pastor, R. W.; Brooks, B. R. J. Chem. Phys. 1995, 103, 4613-4621.

(65) Hoover, W. G. Phys. Rev. A 1985, 31, 1695-1697.

(66) Venable, R. M.; Brooks, B. R.; Pastor, R. W. J. Phys. Chem. 2000, $112,4822-2832$

(67) Nagle, J. F.; Tristram-Nagle, S. Biochim. Biophys. Acta 2000, 1469 , $159-195$.

(68) Pastor, R. W.; Venable, R. M.; Feller, S. E. Acc. Chem. Res. 2002 $35,438-446$

(69) Sundaralingam, M. Ann. N.Y. Acad. Sci. 1972, 195, 324.

(70) Shieh, H. S.; Hoard, L. G.; Nordman, C. E. Acta Crystallogr. 1981, B37, 1538-1543.

(71) Léonard, A.; Escrive, C.; Laguerre, M.; Pebay-Peyroula, E.; Néri,

W.; Pott, T.; Katsaras, J.; Dufourc, E. Langmuir 2001, 17, 2019-2030.

(72) Accelrys. San Diego, CA, 2000.

(73) Jorgensen, W. L.; Chandrasekhar, J.; Madura, J. D.; Impey, R. W.; Klein, M. L. J. Chem. Phys. 1983, 79, 926.

(74) Rog, T.; Pasenkiewicz-Gierula, M. Biophys. J. 2001, 81, 21902202.

(75) Douliez, J.-P.; Léonard, A.; Dufourc, E.-J. Biophys. J. 1995, 68, $1727-1739$

(76) Dufourc, E. J.; Parish, E. J.; Chitrakorn, S.; Smith, I. C. P. Biochemistry 1984, 23, 6062-6071.

(77) Urbina, J. A.; Pekerar, S.; Le, H.; Patterson, J.; Montez, B.; Oldfield, E. Biochim. Biophys. Acta 1995, 1238, 163

(78) Sankaram, M. B.; Thompson, T. E. Biochemistry 1990, 29, 1067610684

(79) Huster, D.; Scheidt, H. A.; Arnold, K.; Hermann, A.; Mueller, P. Biophys. J. 2005, 88, 1838-1844.

(80) Hauser, H.; Pascher, I.; Pearson, R. H.; Sundell, S. Biochim. Biophys. Acta 1981, 650, 21-51.

(81) Mendelsohn, R.; Snyder, R. G. In Biological Membranes: A Molecular Perspective from Computation and Experiment; Birkhäuser: Boston, 1996; pp 145-174.

(82) Casal, H. L.; McElhaney, R. N. Biochemistry 1990, 29, 54235427.

(83) Mendelsohn, R.; Davies, M. A.; Schuster, H. F.; Xu, Z.; Bittman, R. Biochemistry 1991, 30, 8558-8563.

(84) Senak, L.; Moore, D.; Mendelsohn, R. J. Phys. Chem. 1992, 96 2749-2754

(85) Wiener, M. C.; Suter, R. M.; Nagle, J. F. Biophys. J. 1989, 55, $315-325$.

(86) Nagle, J. F.; Zhang, R.; Tristram-Nagle, S.; Sun, W.; Petrache, H. I.; Suter, R. M. Biophys. J. 1996, 70, 1419-1431.

(87) Feller, S. E.; MacKerell, A. D., Jr. J. Phys. Chem. B 2000, 104, $7510-7515$.

(88) Henriksen, J.; Rowat, A. C.; Brief, E.; Hsueh, Y. W.; Thewalt, J. L.; Zuckermann, M. J.; Ipsen, J. H. Biophys. J. 2006, 90, 1639-1649.

(89) Pencer, J.; Nieh, M.-P.; Harroun, T. A.; Krueger, S.; Adams, C.; Katsaras, J. Biochim. Biophys. Acta 2005, 1720, 84-91.

(90) Ipsen, J. H.; Mouritsen, O. G.; Bloom, M. Biophys. J. 1990, 57, 405-412.

(91) Shieh, H. S.; Hoard, L. G.; Nordman, C. E. Nature 1977, 267, $287-$ 289

(92) Craven, B. M. Acta Crystallogr. 1979, B35, 1123-1128.

(93) Hsu, H.-Y.; Kampf, J. W.; Nordman, C. E. Acta Crystallogr. 2002 B58, 260-264.

(94) Baginski, M.; Tempczyk.; Borowski, E. Eur. Biophys. J. 1989, 17, 159-166.

(95) Child, P.; Kuksis, A. J. Lipid Res. 1983, 24, 1196-1209.

(96) Wang, J.; Megha, K.; London, E. Biochemistry 2004, 43, 10101018

(97) Tieleman, D. P.; Berendsen, H. J. C. J. Phys. Chem. 1996, 105, $4871-4880$

(98) de Vries, A. H.; Yefimov, S.; Mark, A. E.; Marrink, S. J. Proc. Natl. Acad. Sci. U.S.A. 2005, 102, 5392-5396. 
(99) de Vries, A. H.; Chandrasekhar, I.; van Gunsteren, W. F.; Hünenberger, P. H. J. Phys. Chem. B 2005, 109, 11643-11652.

(100) Lindahl, E.; Edholm, O. Biophys. J. 2000, 79, 426-433.

(101) Jensen, M. O.; Mouritsen, O. G.; Peters, G. H. Biophys. J. 2004, 86, 3556-3575.

(102) Essmann, U.; Berkowitz, M. L. Biophys. J. 1999, 76, 2081-2089.

(103) Chiu, S. W.; Jakobsson, E.; Subramaniam, S.; Scott, H. L. Biophys.

J. 1999, 77, 2462-2469.

(104) Pasenkiewicz-Gierula, M.; Takaoka, Y.; Miyagawa, H.; Kitamura,

K.; Kusumi, A. J. Phys. Chem. A 1997, 101, 3677-3691.

(105) Tu, K.; Tobias, D. J.; Klein, M. L. Biophys. J. 1995, 69, 25582562 .

(106) Chiu, S.-W.; Clark, M.; Balaki, V.; Subramaniam, S.; Scott, H. L.; Jakobsson, E. Biophys. J. 1995, 69, 1230-1245.

(107) Feller, S. E.; Pastor, R. W. Biophys. J. 1996, 71, 1350-1355.

(108) Marrink, S. J.; Mark, A. E. J. Phys. Chem. B 2001, 105, 61226127.

(109) Skibinksy, A.; Venable, R. M.; Pastor, R. W. Biophys. J. 2005, $89,4111-4121$
(110) Baginski, M.; Resat, H.; McCammon, J. A. Mol. Pharmacol. 1997, $52,560-570$.

(111) Jähnig, F. Biophys. J. 1996, 71, 1348-1349.

(112) Feller, S. E.; Zhang, Y.; Pastor, R. W. J. Chem. Phys. 1995, 103 10267-10276.

(113) Feller, S. E.; Pastor, R. W. J. Chem. Phys. 1999, 111, 12811287.

(114) Sankararamakrishnan, R.; Weinstein, H. J. Phys. Chem. B 2004, 108, 11802-11811.

(115) Patra, M.; Karttunen, M.; Hyvönen, M. T.; Falck, E.; Lindqvist, P.; Vattulainen, I. Biophys. J. 2003, 84, 3636-3645.

(116) Lindahl, E.; Hess, B.; van der Spoel, D. J. Mol. Model. 2001, 7, $306-317$.

(117) Chandrasekhar, I.; Bakowies, D.; Glättli, A.; Hünenberger, P.; Pereira, C.; van Gunsteren, W. F. Mol. Simul. 2005, 31, 543-548.

(118) Nagle, J. F.; Wiener, M. C. Biochim. Biophys. Acta 1988, 942 $1-10$.

(119) Tristram-Nagle, S.; Zhang, R.; Suter, R. M.; Worthington, C. R.; Sun, W. J.; Nagle, J. F. Biophys. J. 1993, 64, 1097. 